\title{
ON SEPARABLE HIGHER GAUSS MAPS
}

\author{
KATSUHISA FURUKAWA AND ATSUSHI ITO
}

\begin{abstract}
We study the $m$-th Gauss map in the sense of F. L. Zak of a projective variety $X \subset \mathbb{P}^{N}$ over an algebraically closed field in any characteristic. For all integer $m$ with $n:=\operatorname{dim}(X) \leqslant m<N$, we show that the contact locus on $X$ of a general tangent $m$-plane is a linear variety if the $m$-th Gauss map is separable. We also show that for smooth $X$ with $n<N-2$, the $(n+1)$ th Gauss map is birational if it is separable, unless $X$ is the Segre embedding $\mathbb{P}^{1} \times \mathbb{P}^{n} \subset \mathbb{P}^{2 n-1}$. This is related to L. Ein's classification of varieties with small dual varieties in characteristic zero.
\end{abstract}

\section{INTRODUCTION}

Let $X \subset \mathbb{P}^{N}$ be an $n$-dimensional non-degenerate projective variety over an algebraically closed field in characteristic $p \geqslant 0$, and let $m$ be an integer with $n \leqslant m<N$. We define the $m$-th Gauss map $\gamma_{m}$,

$$
\gamma_{m}: P_{m}^{X}:=\overline{\left\{(L, x) \in \mathbb{G}\left(m, \mathbb{P}^{N}\right) \times X^{s m} \mid \mathbb{T}_{x} X \subset L\right\}} \rightarrow \mathbb{G}\left(m, \mathbb{P}^{N}\right),
$$

to be the first projection from the incidence $P_{m}^{X}$ to the Grassmann variety $\mathbb{G}\left(m, \mathbb{P}^{N}\right)$, where $X^{s m}$ is the smooth locus of $X$, and $\mathbb{T}_{x} X \subset \mathbb{P}^{N}$ is the embedded tangent space to $X$ at $x$. We set $X_{m}^{*}=\gamma_{m}\left(P_{m}^{X}\right) \subset \mathbb{G}\left(m, \mathbb{P}^{N}\right)$, the set of $m$-planes tangent to $X$. See $[29, \mathrm{I}, \S 2]$.

Note that $\gamma_{n}$ is identified with the (ordinary) Gauss map $X-\rightarrow \mathbb{G}\left(n, \mathbb{P}^{N}\right): x \mapsto$ $\mathbb{T}_{x} X$, and $X_{N-1}^{*}$ is the projective dual variety $X^{*} \subset\left(\mathbb{P}^{N}\right)^{\vee}$ of $X$. For a tangent $m$-plane $L \in X_{m}^{*}$, we consider the contact locus on $X$ of $L$,

$$
\overline{\left\{x \in X^{s m} \mid \mathbb{T}_{x} X \subset L\right\}},
$$

which is equal to $\pi\left(\gamma_{m}^{-1}(L)_{r e d}\right) \subset X$ if $L \in X_{m}^{*}$ is general, where $\pi: P_{m}^{X} \rightarrow X$ is the second projection. It is also called an $m$-th contact locus, and is said to be general if so is $L$.

In characteristic $p=0$, it was well know that a general $m$-th contact locus is a linear variety of $\mathbb{P}^{N}$ for all $m$ with $n \leqslant m<N$; the case of $m=n$ is the linearity of a general fiber of the Gauss map (P. Griffiths and J. Harris [13, (2.10)],

2010 Mathematics Subject Classification. 14 N05.

Key words and phrases. higher Gauss map, linearity of a general contact locus, dual defect. 
F. L. Zak [29, I, 2.3. Theorem (c)]) and the case of any $m$ can be shown due to the reflexivity (S. L. Kleiman and R. Piene [22, pp. 108-109]). H. Kaji [19] recently gave a formula for the degree of $X_{m}^{*}$ in the case when $\gamma_{m}$ is generically finite, in particular, the case when $X=v_{d}\left(\mathbb{P}^{n}\right) \subset \mathbb{P}^{N}$ is the Veronese variety with $N=\left(\begin{array}{c}n+d \\ d\end{array}\right)$ as a generalization of Boole's formula for $\operatorname{deg}\left(X^{*}\right)$.

In characteristic $p>0$, the linearity of a general contact locus does not hold in general; in particular for $m=n$, several authors gave examples of $X$ such that a general fiber of $\gamma_{n}$ is not a linear variety ([28, §7], [21, I-3], [16, Example 4.1], [17], [27, Example 2.13], [24], [6, §7], [7], [11, §5], [12, Theorem 1.3]).

However, it was known that a general $m$-th contact locus is a linear variety of $\mathbb{P}^{N}$ if $\gamma_{m}$ is separable in the case when $m=N-1$ (by the Monge-Segre-Wallace criterion $[14,(2.4)],[21, \mathrm{I}-1(4)])$, and in the case when $m=n$ (the first author $[10])$.

In this paper, we prove the following theorem for all $m$.

Theorem 1.1. Let $X \subset \mathbb{P}^{N}$ be a projective variety in characteristic $p \geqslant 0$, and let $m$ be an integer with $n=\operatorname{dim}(X) \leqslant m<N$. Assume that the $m$-th Gauss map $\gamma_{m}$ is separable. Then a general $m$-th contact locus (1.1) is a linear variety of $\mathbb{P}^{N}$.

If $\gamma_{m}$ is separable, then so is $\gamma_{m^{\prime}}$ for all $m^{\prime}$ with $n \leqslant m^{\prime} \leqslant m$; this can be shown in the same way for the reflexive case. See Remark 2.3. On the other hand, for $m^{\prime \prime}>m, \gamma_{m^{\prime \prime}}$ can be inseparable even if $\gamma_{m}$ is separable; H. Kaji [18] and S. Fukasawa [8] [9] gave such examples for the case $m^{\prime \prime}=N-1$ and $m=n$.

F. L. Zak showed that a contact locus of any $L \in \gamma_{m}\left(\pi^{-1}\left(X^{s m}\right)\right)$ is of dimension $\leqslant m-n+\operatorname{dim}(\operatorname{Sing}(X))+1[29$, I. $\S 2$, Theorem 2.3(a)]. Hence, if $X$ is smooth, then any $m$-th contact locus is of dimension $\leqslant m-n$. Considering the case of $m=n$ and combining with the linearity (in particular, irreducibility) of a general fiber of the $n$-th Gauss map $\gamma_{n}$, it holds that $\gamma_{n}$ is in fact birational if it is separable (e.g., the characteristic $p=0$ ).

In the case $m=n+1$, any $(n+1)$-th contact locus is of dimension $\leqslant 1$ if $X$ is smooth. We study when the equality holds, and obtain the following theorem.

Theorem 1.2. Let $X \subset \mathbb{P}^{N}$ be a smooth non-degenerate projective variety in characteristic $p \geqslant 0$ such that $n=\operatorname{dim}(X)<N-2$ and $\gamma_{n+1}$ is separable. Then $\gamma_{n+1}$ is in fact birational, unless $X$ is the Segre embedding $\mathbb{P}^{1} \times \mathbb{P}^{n-1} \subset \mathbb{P}^{2 n-1}$.

In characteristic $p=0$, since the case of $n=N-2$ follows from [3, Theorem 3.4], the statement holds for all $n \leqslant N-2$, and we have that $\mathbb{P}^{1} \times \mathbb{P}^{n-1} \subset \mathbb{P}^{2 n-1}$ is only the smooth projective variety which satisfies the equality for Zak's inequality on dimensions of $m$-th contact loci for any $n \leqslant m<N$. See Theorem 4.8 and Remark 4.9 for detail. 
Note that if $\gamma_{n+1}$ is separable and generically finite, then it is birational because of Theorem 1.1. Hence the main point of the proof of Theorem 1.2 is to study the case when $\gamma_{n+1}$ is not generically finite.

A background of our study is the classification of varieties with small dual varieties by L. Ein [3]; in characteristic $p=0$, he showed that if a smooth projective variety $X \subset \mathbb{P}^{N}$ satisfies $\operatorname{dim}(X)=\operatorname{dim}\left(X^{*}\right) \leqslant \frac{2}{3} N$, then $X$ is one of the followings; (a) a hypersurface, (b) $\mathbb{P}^{1} \times \mathbb{P}^{n-1} \subset \mathbb{P}^{2 n-1}$, (c) $\mathbb{G}\left(1, \mathbb{P}^{4}\right) \subset \mathbb{P}^{9}$, (d) the 10 -dimensional spinor variety in $\mathbb{P}^{15}$. We note that the Ein's classification is completed if Hartshorne's conjecture holds, that is to say, any smooth projective variety $X \subset \mathbb{P}^{N}$ is a complete intersection if $\operatorname{dim}(X)>\frac{2}{3} N$.

If $\gamma_{n+1}$ is not generically finite, then we have $\operatorname{dim}(X)=\operatorname{dim}\left(X^{*}\right)$ (see Corollary 3.4). On the other hand, if $X$ is (c) or (d), then the finiteness of $\gamma_{n+1}$ can be shown by explicit calculations. Hence we find that the statement of Theorem 1.2 follows from Ein's result in characteristic $p=0$ if $\operatorname{dim}(X) \leqslant \frac{2}{3} N$ (or Hartshorne's conjecture holds). Our purpose is to show this statement in any characteristic $p \geqslant 0$ without the condition of the dimension of $X$.

This paper is organized as follows. In $\S 2$, we generalize the techniques of [10] and [12] in order to study $m$-th Gauss maps in arbitrary characteristic. In particular, we define and describe the $m$-th degeneracy map and prove Theorem 1.1. In $\S 3$, we show basic properties of $m$-th contact loci and their dimensions, i.e., $m$-th defects. In $\S 4$, we study the $(n+1)$-th Gauss map $\gamma_{n+1}$ in the case when $X$ is smooth and $\gamma_{n+1}$ is not generically finite. We fix a general point $x \in X$ and consider the union of contact loci of $(n+1)$-planes $L$ 's such that $L$ is tangent to $X$ at $x$. Showing that the union is in fact an $(n-1)$-plane, we have that $X$ is the Segre embedding $\mathbb{P}^{1} \times \mathbb{P}^{n-1}$, yielding Theorem 1.2.

Acknowledgments. The authors would like to thank Professors Satoru Fukasawa and Hajime Kaji for their valuable comments and advice. The first author was supported by the Grant-in-Aid for JSPS fellows, No. 16J00404. The second author was supported by the Grant-in-Aid for JSPS fellows, No. 26-1881.

\section{LineARITY OF GENERAL $m$-TH CONTACT LOCI}

In [12, Definition 2.1], we extended the notion of the shrinking map, which had been independently introduced by J. M. Landsberg and J. Piontkowski (see [5, 2.4.7] and [15, Theorem 3.4.8]). Here we recall the definition of the map, which is a main ingredient of this section.

Definition 2.1. We denote by $\mathcal{S}=\mathcal{S}_{m}$ and $\mathcal{Q}=Q_{m}$ the universal subbundle of rank $N-m$ and the universal quotient bundle of rank $m+1$ over $\mathbb{G}\left(m, \mathbb{P}^{N}\right)$ 
respectively, with the exact sequence

$$
0 \rightarrow \mathcal{S} \rightarrow H^{0}\left(\mathbb{P}^{N}, \mathcal{O}(1)\right) \otimes \mathcal{O}_{\mathbb{G}\left(m, \mathbb{P}^{N}\right)} \rightarrow \mathcal{Q} \rightarrow 0 .
$$

For a rational map $f: Z \rightarrow \mathbb{G}\left(m, \mathbb{P}^{N}\right)$, we define the shrinking map of $Z$ with respect to $f$

$$
\sigma=\sigma_{Z, f}: Z \rightarrow \mathbb{G}\left(m^{-}, \mathbb{P}^{N}\right)
$$

for some integer $m^{-}=m_{\sigma}^{-} \leqslant m$ as follows. Let $Z^{\circ}$ be an open subset consisting of smooth points of $Z$ and regard $f$ as $\left.f\right|_{Z^{\circ}}$. We have the following composite homomorphism

$$
\Phi=\Phi_{f}: f^{*} \mathcal{Q}^{\vee} \rightarrow f^{*} \mathcal{H} \operatorname{com}\left(\mathcal{H} \operatorname{Com}\left(\mathcal{Q}^{\vee}, \mathcal{S}^{\vee}\right), \mathcal{S}^{\vee}\right) \rightarrow \mathcal{H} \operatorname{Com}\left(T_{Z^{\circ}}, f^{*} \mathcal{S}^{\vee}\right),
$$

where the first homomorphism is induced from the dual of $\mathcal{S} \otimes \mathcal{S}^{\vee} \rightarrow \mathcal{O}$, and the second one is induced from the differential $d f: T_{Z^{\circ}} \rightarrow f^{*} T_{\mathbb{G}\left(n, \mathbb{P}^{N}\right)}=f^{*} \mathcal{H} \operatorname{Com}\left(\mathcal{Q}^{\vee}, \mathcal{S}^{\vee}\right)$. We define an integer $m^{-}$with $-1 \leqslant m^{-} \leqslant m$ by

$$
m^{-}=\operatorname{dim}(\operatorname{ker} \Phi \otimes k(z))-1
$$

for a general point $z \in Z$. Since ker $\left.\Phi\right|_{Z^{\circ}}$ is a subbundle of $H^{0}\left(\mathbb{P}^{N}, \mathcal{O}(1)\right)^{\vee} \otimes \mathcal{O}_{Z^{\circ}}$ of rank $m^{-}+1$ (replacing $Z^{\circ} \subset Z$ by a smaller open subset if necessary), we have an induced morphism $\sigma: Z^{\circ} \rightarrow \mathbb{G}\left(m^{-}, \mathbb{P}^{N}\right)$ and call it the shrinking map of $Z$ with respect to $f$.

The purpose of this section is to prove the following result, where we write

$$
\mathcal{U}_{\mathbb{G}\left(m, \mathbb{P}^{N}\right)}:=\left\{(L, x) \in \mathbb{G}\left(m, \mathbb{P}^{N}\right) \times \mathbb{P}^{N} \mid x \in L\right\},
$$

the universal family of $\mathbb{G}\left(m, \mathbb{P}^{N}\right)$.

Theorem 2.2. Let $X \subset \mathbb{P}^{N}$ be an $n$-dimensional projective variety, and let $\iota$ : $Y \hookrightarrow \mathbb{G}\left(m, \mathbb{P}^{N}\right)$ be a projective variety with $n \leqslant m \leqslant N-1$. We set $\sigma_{Y}=$ $\sigma_{Y, \iota}: Y \rightarrow \mathbb{G}\left(m^{-}, \mathbb{P}^{N}\right)$ to be the shrinking map of $Y$ with respect to $\iota$, where $m^{-}=m_{\sigma_{Y}}^{-} \leqslant m$. Then the following are equivalent:

(1) $\gamma_{m}: P_{m}^{X} \rightarrow \mathbb{G}\left(m, \mathbb{P}^{N}\right)$ is separable, and $Y=\gamma_{m}\left(P_{m}^{X}\right)$.

(2) $P_{m}^{X}=\sigma_{Y}^{*} \mathcal{U}_{\mathbb{G}\left(m^{-}, \mathbb{P}^{N}\right)}$ in $\mathbb{G}\left(m, \mathbb{P}^{N}\right) \times \mathbb{P}^{N}$.

(3) $\operatorname{dim} \sigma_{Y}^{*} \mathcal{U}_{\mathbb{G}\left(m^{-}, \mathbb{P}^{N}\right)}=n+(m-n)(N-m)$ and the second projection $\sigma_{Y}^{*} \mathcal{U}_{\mathbb{G}\left(m^{-}, \mathbb{P}^{N}\right)} \rightarrow$ $\mathbb{P}^{N}$ is separable, and the image is equal to $X$.

Once the above theorem is shown, Theorem 1.1 follows from the implication "(1) $\Rightarrow(2) "$ as follows.

Proof of Theorem 1.1. Take a general $m$-plane $[L] \in X_{m}^{*}=\gamma_{m}\left(P_{m}^{X}\right)=Y$ and set $M \subset \mathbb{P}^{N}$ to be the $m^{-}$-plane corresponding to $\sigma_{Y}(L) \in \mathbb{G}\left(m^{-}, \mathbb{P}^{N}\right)$. By Theorem $2.2(2)$, we have $\gamma_{m}^{-1}(L)=\{[L]\} \times M$. Hence it holds that $\pi\left(\gamma_{m}^{-1}(L)\right)=M$.

Note that we also have a characterization of images of separable $m$-th Gauss maps by the equivalence "(1) $\Leftrightarrow(3)$ " (see [10, Corollary 3.15]). 
Remark 2.3. If $\gamma_{m}$ is separable, then so is $\gamma_{m^{\prime}}$ for all $m^{\prime}$ with $n \leqslant m^{\prime} \leqslant m$. This is shown as follows, which is the same way for the reflexive case by Kleiman and Piene [22, pp. 108-109]. It is sufficient to show the case $m^{\prime}=m-1$. Consider the Flag variety

$$
\mathbb{F}\left(m-1, m ; \mathbb{P}^{N}\right)=\left\{\left(M^{\prime}, M\right) \in \mathbb{G}\left(m-1, \mathbb{P}^{N}\right) \times \mathbb{G}\left(m, \mathbb{P}^{N}\right) \mid M^{\prime} \subset M\right\},
$$

and define $P_{m-1, m}^{X}$ to be the closure of the set of $\left(M^{\prime}, M, x\right) \in \mathbb{F}\left(m-1, m ; \mathbb{P}^{N}\right) \times X^{s m}$ such that $\mathbb{T}_{x} X \subset M^{\prime} \subset M$. We have the following commutative diagram:

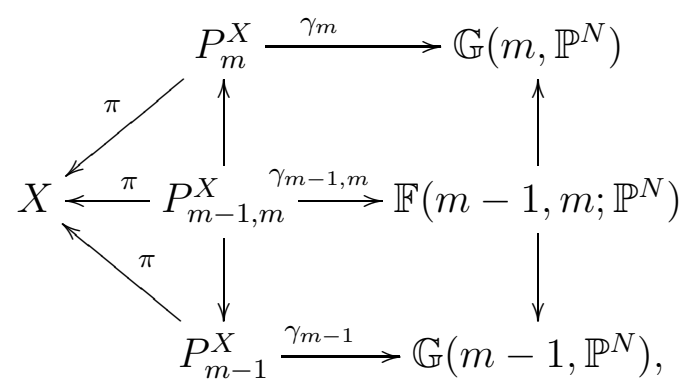

where $\pi\left(\gamma_{m-1, m}^{-1}\left(M^{\prime}, M\right)\right)=\pi\left(\gamma_{m-1}^{-1}\left(M^{\prime}\right)\right)$ in $X$ for $\left(M^{\prime}, M\right) \in \mathbb{F}\left(m-1, m ; \mathbb{P}^{N}\right)$. By the diagram, $\pi\left(\gamma_{m-1}^{-1}\left(M^{\prime}\right)\right) \subset X$ is scheme-theoretically equal to the intersection of $\pi\left(\gamma_{m}^{-1}(M)\right)$ 's with general $m$-planes $M$ containing $M^{\prime}$. If $\gamma_{m}$ is separable, then Theorem 1.1 implies that $\pi\left(\gamma_{m}^{-1}(M)\right)$ is a linear variety, and hence so is $\pi\left(\gamma_{m-1}^{-1}\left(M^{\prime}\right)\right)$. In particular, $\pi\left(\gamma_{m-1}^{-1}\left(M^{\prime}\right)\right) \simeq \gamma_{m-1}^{-1}\left(M^{\prime}\right)$ is reduced, that is to say $\gamma_{m-1}$ is separable.

The case of $m=n(=\operatorname{dim}(X))$ in Theorem 2.2 is nothing but [10, Theorem 3.1], [12, Corollary 5.4]. In order to prove Theorem 2.2 for any $m \geqslant n$, we generalize several techniques of [12] in the following subsections.

2.1. Subvarieties of the universal family of a Grassmann variety. We set the bundles $\mathcal{S}=\mathcal{S}_{m}$ and $\mathcal{Q}=\mathcal{Q}_{m}$ on $\mathbb{G}\left(m, \mathbb{P}^{N}\right)$ as in Definition 2.1. Set $\mathcal{U}=$ $\mathcal{U}_{\mathbb{G}\left(m, \mathbb{P}^{N}\right)}$ to be the universal family of $\mathbb{G}\left(m, \mathbb{P}^{N}\right)$. Then $P_{m}^{X} \subset \mathcal{U}$ holds for a projective variety $X \subset \mathbb{P}^{N}$. We denote by $\mathcal{O}_{\mathcal{U}}(1)$ the tautological invertible sheaf on $\mathcal{U}=\mathbb{P}_{*}\left(Q^{\vee}\right)$.

First we give equivalent conditions when a subvariety of $\mathcal{U}$ is contained in $P_{m}^{X}$ for some $X$.

Proposition 2.4. Let $X^{\prime} \subset \mathcal{U} \subset \mathbb{G}\left(m, \mathbb{P}^{N}\right) \times \mathbb{P}^{N}$ be a projective variety and let $\mathrm{pr}_{i}$ be the projection from $X^{\prime}$ to the $i$-th factor for $i=1,2$. For simplicity, we also denote by $\mathrm{pr}_{i}$ the restricted morphism $\left.\mathrm{pr}_{i}\right|_{X^{\prime}}$ for a non-empty open subset $X^{\prime \circ} \subset X^{\prime s m}$. Let $\Phi=\Phi_{\mathrm{pr}_{1}}: \operatorname{pr}_{1}^{*} \mathcal{Q}^{\vee} \rightarrow \mathcal{H} \operatorname{Hom}\left(T_{X^{\prime \circ}}, \operatorname{pr}_{1}^{*} \mathcal{S}^{\vee}\right)$ and $\sigma=\sigma_{X^{\prime}, \mathrm{pr}_{1}}: X^{\prime} \rightarrow$ $\mathbb{G}\left(m^{-}, \mathbb{P}^{N}\right)$ be as in Definition 2.1.

Assume that $\mathrm{pr}_{2}: X^{\prime} \rightarrow \mathbb{P}^{N}$ is separable. Then the following conditions are equivalent. 
(i) $X^{\prime} \subset P_{m}^{X}$ for $X=\operatorname{pr}_{2}\left(X^{\prime}\right)$.

(ii) The composition of

$$
\left.\mathcal{O}_{\mathcal{U}}(-1)\right|_{X^{\prime \circ}} \hookrightarrow \operatorname{pr}_{1}^{*} \mathcal{Q}^{\vee} \text { and } \Phi: \operatorname{pr}_{1}^{*} \mathcal{Q}^{\vee} \rightarrow \mathcal{H} \operatorname{Hom}\left(T_{X^{\prime \circ}}, \operatorname{pr}_{1}^{*} \mathcal{S}^{\vee}\right)
$$

is the zero map on a non-empty open subset $X^{\prime \circ} \subset X^{\prime}$.

(iii) The image of $\left(\sigma, \mathrm{pr}_{2}\right): X^{\prime} \rightarrow \mathbb{G}\left(m^{-}, \mathbb{P}^{N}\right) \times \mathbb{P}^{N}$ is contained in the universal family $\mathcal{U}_{\mathbb{G}\left(m^{-}, \mathbb{P}^{N}\right)} \subset \mathbb{G}\left(m^{-}, \mathbb{P}^{N}\right) \times \mathbb{P}^{N}$.

Proof. On $\mathcal{U}$, there exists a natural surjection $\varepsilon: \overline{\mathrm{pr}}_{2}^{*} T_{\mathbb{P}^{N}}(-1) \rightarrow \overline{\mathrm{pr}}_{1}^{*} \mathcal{S}^{\vee}$, where $\overline{\mathrm{pr}}_{i}$ is the projection from $\mathcal{U} \subset \mathbb{G}\left(m, \mathbb{P}^{N}\right) \times \mathbb{P}^{N}$ to the $i$-th factor (hence $\operatorname{pr}_{i}=\left.\overline{\operatorname{pr}}_{i}\right|_{X^{\prime}}$ ). We note that $\mathcal{O}_{\mathcal{U}}(1)=\overline{\operatorname{pr}}_{2}^{*} \mathcal{O}_{\mathbb{P}^{N}}(1)$ holds.

Set $X=\operatorname{pr}_{2}\left(X^{\prime}\right)$. Since $P_{m}^{X}$ is the incidence variety, $X^{\prime}$ is contained in $P_{m}^{X}$ if and only if

$$
\operatorname{pr}_{2}^{*} T_{X^{s m}}(-1) \hookrightarrow \operatorname{pr}_{2}^{*} T_{\mathbb{P}^{N}}(-1) \rightarrow \operatorname{pr}_{1}^{*} \mathcal{S}^{\vee}
$$

is the zero map on $X^{\prime \circ}$. By the separability of the dominant morphism $\mathrm{pr}_{2}$ : $X^{\prime \circ} \rightarrow X \subset \mathbb{P}^{N}, d\left(\mathrm{pr}_{2}\right): T_{X^{\prime \circ}} \rightarrow \operatorname{pr}_{2}^{*} T_{X^{s m}}$ is surjective (by taking smaller $X^{\prime 0}$ if necessary). Hence the above map is the zero map if and only if so is

$$
T_{X^{\prime \circ}}(-1) \stackrel{d\left(\mathrm{pr}_{2}\right)(-1)}{\longrightarrow} \operatorname{pr}_{2}^{*} T_{X^{s m}}(-1) \hookrightarrow \mathrm{pr}_{2}^{*} T_{\mathbb{P}^{N}}(-1) \rightarrow \mathrm{pr}_{1}^{*} \mathcal{S}^{\vee}
$$

By the same argument as in the proof of (i) $\Leftrightarrow$ (ii) in [12, Theorem 2.4], we see that (2.2) corresponds to

$$
\left.\mathcal{O}_{\mathcal{U}}(-1)\right|_{X^{\prime \circ}} \hookrightarrow \operatorname{pr}_{1}^{*} \mathcal{Q}^{\vee} \stackrel{\Phi}{\longrightarrow} \mathcal{H} \operatorname{Hom}\left(T_{X^{\prime \circ}}, \operatorname{pr}_{1}^{*} \mathcal{S}^{\vee}\right) .
$$

under the identification

$$
\mathcal{H} \operatorname{Hom}\left(T_{X^{\prime \circ}}(-1), \operatorname{pr}_{1}^{*} \mathcal{S}^{\vee}\right)=\mathcal{H} \operatorname{com}\left(\left.\mathcal{O}_{\mathcal{U}}(-1)\right|_{X^{\prime \circ}}, \mathcal{H} \operatorname{Hom}\left(T_{X^{\prime \circ}}, \operatorname{pr}_{1}^{*} \mathcal{S}^{\vee}\right)\right) \text {. }
$$

Thus (i) and (ii) are equivalent. The proof of the equivalence of (ii) and (iii) is the same as that of $[12$, Theorem 2.4]

2.2. Shrinking maps and $m$-th degeneracy maps. We define the $m$-th degeneracy map as follows, which will play a key role in the proof of Theorem 2.2.

Definition 2.5. Let $X \subset \mathbb{P}^{N}$ be an $n$-dimensional projective variety and $X^{\circ} \subset$ $X^{s m}$ be a non-empty open subset. Set $P_{m}^{X^{\circ}}:=P_{m}^{X} \cap\left(\mathbb{G}\left(m, \mathbb{P}^{N}\right) \times X^{\circ}\right)$.

Take $d \gamma_{m}: T_{P_{m}^{X \circ}} \rightarrow \gamma_{m}^{*} T_{\mathbb{G}\left(m, \mathbb{P}^{N}\right)}$ and $d \pi: T_{P_{m}^{X \circ}} \rightarrow \pi^{*} T_{X^{s m}}$ to be the differentials of the $m$-th Gauss map $\gamma_{m}$ and the projection $\pi: P_{m}^{X} \rightarrow X \subset \mathbb{P}^{N}$. Then $d \pi\left(\operatorname{ker}\left(d \gamma_{m}\right)\right) \subset \pi^{*} T_{X^{s m}} \subset \pi^{*} T_{\mathbb{P}^{N}}$ is isomorphic to $\operatorname{ker}\left(d \gamma_{m}\right)$ since $T_{P_{m}^{X}}$ is contained in $\left.T_{\mathbb{G}\left(m, \mathbb{P}^{N}\right) \times \mathbb{P}^{N}}\right|_{P_{m}^{X}}=\gamma_{m}^{*} T_{\mathbb{G}\left(m, \mathbb{P}^{N}\right)} \oplus \pi^{*} T_{\mathbb{P}^{N}}$.

Set $n_{\kappa_{m}}^{-}$to be the rank of the torsion free sheaf $\operatorname{ker}\left(d \gamma_{m}\right)$. Pulling back the Euler sequence

$$
0 \rightarrow \mathcal{O}_{\mathbb{P}^{N}} \rightarrow H^{0}\left(\mathbb{P}^{N}, \mathcal{O}(1)\right)^{\vee} \otimes \mathcal{O}_{\mathbb{P}^{N}}(1) \rightarrow T_{\mathbb{P}^{N}} \rightarrow 0
$$


we have $\theta: H^{0}\left(\mathbb{P}^{N}, \mathcal{O}(1)\right)^{\vee} \otimes \mathcal{O}_{P_{m}^{X}} \rightarrow \pi^{*} T_{\mathbb{P}^{N}}(-1)$. Then the subsheaf

$$
\theta^{-1}\left(d \pi\left(\operatorname{ker}\left(d \gamma_{m}\right)\right)(-1)\right) \subset H^{0}\left(\mathbb{P}^{N}, \mathcal{O}(1)\right)^{\vee} \otimes \mathcal{O}_{P_{m}^{X}} \circ
$$

induces a rational map

$$
\kappa_{m}: P_{m}^{X} \rightarrow \mathbb{G}\left(n_{\kappa_{m}}^{-}, \mathbb{P}^{N}\right),
$$

which we call the $m$-th degeneracy map of $X$. In the case of $m=n, \kappa_{n}$ can be identified with the usual degeneracy map under the birational map $P_{n}^{X} \rightarrow X$.

The rest of this section is devoted to the proof of the following proposition, which is a generalization of [12, Proposition 5.2].

Proposition 2.6. Let $X \subset \mathbb{P}^{N}$ be a projective variety. Then the $m$-th degeneracy map $\kappa_{m}$ coincides with the shrinking map $\sigma_{P_{m}^{X}, \gamma_{m}}$ of $P_{m}^{X}$ with respect to $\gamma_{m}: P_{m}^{X} \rightarrow$ $\mathbb{G}\left(m, \mathbb{P}^{N}\right)$.

First we recall the definition of the second fundamental form $\tau$. Let $\mathcal{S}_{n}$ and $Q_{n}$ be the universal subbundle and the universal quotient bundle over $\mathbb{G}\left(n, \mathbb{P}^{N}\right)$. Considering the $n$-th Gauss map (ordinary Gauss map) $\gamma_{n}: X^{\circ} \rightarrow \mathbb{G}\left(n, \mathbb{P}^{N}\right)$ and considering the dual of the sequence (2.1) for $m=n$, we have the following commutative diagram with exact rows and columns,

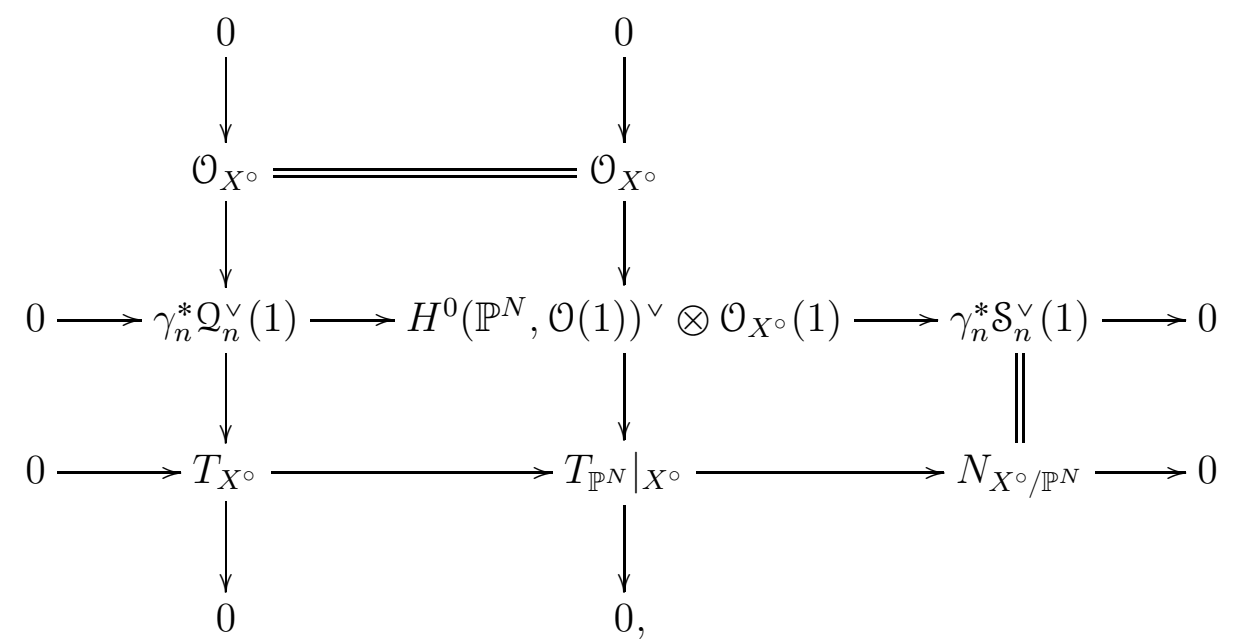

where the middle vertical sequence is induced from the Euler sequence on $\mathbb{P}^{N}$. The differential $d \gamma_{n}: T_{X^{\circ}} \rightarrow \gamma_{n}^{*} T_{\mathbb{G}\left(n, \mathbb{P}^{N}\right)}=\gamma_{n}^{*} \mathcal{H} \operatorname{Com}\left(Q_{n}^{\vee}, \mathcal{S}_{n}^{\vee}\right)$ induces a homomorphism

$$
\widetilde{d \gamma_{n}}: T_{X^{\circ}} \otimes \gamma_{n}^{*} Q_{n}^{\vee} \rightarrow \gamma_{n}^{*} \mathcal{S}_{n}^{\vee}=N_{X^{\circ} / \mathbb{P}^{N}}(-1)
$$

Then we can check that, the composition of $T_{X^{\circ}} \otimes \mathcal{O}_{X^{\circ}} \hookrightarrow T_{X^{\circ}} \otimes \gamma_{n}^{*} Q_{n}^{\vee}(1)$ induced by the first vertical sequence of $(2.3)$ and $\widetilde{d \gamma_{n}}(1): T_{X^{\circ}} \otimes \gamma_{n}^{*} Q_{n}^{\vee}(1) \rightarrow N_{X^{\circ} / \mathbb{P}^{N}}$ is the zero map. Hence a homomorphism, called the second fundamental form,

$$
\tau: T_{X^{\circ}} \otimes T_{X^{\circ}} \rightarrow N_{X^{\circ} / \mathbb{P}^{N}}
$$


is induced. By definition, $\widetilde{d \gamma_{n}}(1)$ factors through $\tau$. In other words, $d \gamma_{n}$ factors as

$$
T_{X^{\circ}} \rightarrow \mathcal{H} \operatorname{Hom}\left(T_{X^{\circ}}(-1), \gamma_{n}^{*} \mathcal{S}_{n}^{\vee}\right) \hookrightarrow \gamma_{n}^{*} \mathcal{H} \operatorname{Hom}\left(Q_{n}^{\vee}, \mathcal{S}_{n}^{\vee}\right),
$$

where the inclusion is induced by the surjection $\gamma_{n}^{*} Q_{n}^{\vee} \rightarrow T_{X^{\circ}}(-1)$. It is known that $\tau$ is symmetric.

Next we consider the following homomorphism $\varphi$. Set $P_{m}^{X^{\circ}}=P_{m}^{X} \cap\left(\mathbb{G}\left(m, \mathbb{P}^{N}\right) \times\right.$ $X^{\circ}$ ) as before. In this subsection, we hereafter write $\operatorname{pr}_{i}$ to be the projection from $P_{m}^{X} \subset \mathbb{G}\left(m, \mathbb{P}^{N}\right) \times X$ to the $i$-the factor. Indeed, $\mathrm{pr}_{1}$ and $\mathrm{pr}_{2}$ are nothing but " $\gamma_{m}$ " and " $\pi$ ", respectively. But we use the symbol " $\mathrm{pr}_{i}$ " to suit the notation of [12]. For simplicity of notation, we also use $\mathrm{pr}_{i}$ for the restricted morphism $\left.\mathrm{pr}_{i}\right|_{P_{m}^{X}}$.

Write $\mathcal{S}=\mathcal{S}_{m}$ and $\mathcal{Q}=\mathcal{Q}_{m}$. Pulling back $\tau \otimes \mathcal{O}(-1)$ by $\mathrm{pr}_{2}: P_{m}^{X} \rightarrow X$ and composing with the natural homomorphism $\operatorname{pr}_{2}^{*} N_{X^{\circ} / \mathbb{P}^{N}}(-1) \rightarrow \operatorname{pr}_{1}^{*} \mathcal{S}^{\vee}$, we have the homomorphism on $P_{m}^{X^{\circ}}$ :

$$
\varphi: \operatorname{pr}_{2}^{*}\left(T_{X^{\circ}} \otimes T_{X^{\circ}}(-1)\right) \rightarrow \operatorname{pr}_{2}^{*} N_{X^{\circ} / \mathbb{P}^{N}}(-1) \rightarrow \operatorname{pr}_{1}^{*} \mathcal{S}^{\vee} .
$$

We define $\varphi_{i}: \operatorname{pr}_{2}^{*} T_{X^{\circ}} \rightarrow \mathcal{H o m}\left(\operatorname{pr}_{2}^{*} T_{X \circ}(-1), \operatorname{pr}_{1}^{*} \mathcal{S}^{\vee}\right)$ for $i=1,2$ by

$$
\begin{aligned}
& \varphi_{1}(x)=\left[\operatorname{pr}_{2}^{*} T_{X^{\circ}}(-1) \rightarrow \operatorname{pr}_{1}^{*} \mathcal{S}^{\vee}: y \mapsto \varphi(x, y)\right], \\
& \varphi_{2}(x)=\left[\operatorname{pr}_{2}^{*} T_{X^{\circ}}(-1) \rightarrow \operatorname{pr}_{1}^{*} \mathcal{S}^{\vee}: y \mapsto \varphi(y, x)\right] .
\end{aligned}
$$

We sometimes regard $\varphi_{i}$ as a homomorphism

$$
\operatorname{pr}_{2}^{*} T_{X^{\circ}} \rightarrow \mathcal{H} \operatorname{Hom}\left(\operatorname{pr}_{2}^{*} \gamma_{n}^{*} Q_{n}^{\vee}, \operatorname{pr}_{1}^{*} \mathcal{S}^{\vee}\right)
$$

by the natural surjection $\gamma_{n}^{*} Q_{n}^{\vee} \rightarrow T_{X^{\circ}}(-1)$. By definition, $\varphi_{1}$ is the homomorphism induced from $\operatorname{pr}_{2}^{*} d \gamma_{n}: \operatorname{pr}_{2}^{*} T_{X^{\circ}} \rightarrow \operatorname{pr}_{2}^{*} \gamma_{n}^{*} \mathcal{H} \operatorname{Lom}\left(Q_{n}^{\vee}, \mathcal{S}_{n}^{\vee}\right)$ and $\operatorname{pr}_{2}^{*} \gamma_{n}^{*} \mathcal{S}_{n}^{\vee}=$ $\operatorname{pr}_{2}^{*} N_{X^{\circ} / \mathbb{P}^{N}}(-1) \rightarrow \operatorname{pr}_{1}^{*} \mathcal{S}^{\vee}$. Furthermore, $\varphi_{1}=\varphi_{2}$ holds because of the symmetry of $\tau$.

We have the following lemma in a general setting.

Lemma 2.7. Let $n \leqslant m<N$ be non-negative integers and let $\mathbb{F}\left(n, m ; \mathbb{P}^{N}\right) \subset$ $\mathbb{G}\left(n, \mathbb{P}^{N}\right) \times \mathbb{G}\left(m, \mathbb{P}^{N}\right)$ be the frag variety parametrizing linear subvarieties $\mathbb{P}^{n} \subset \mathbb{P}^{m}$ in $\mathbb{P}^{N}$. Let $q_{n}: \mathbb{F}\left(n, m ; \mathbb{P}^{N}\right) \rightarrow \mathbb{G}\left(n, \mathbb{P}^{N}\right)$ and $q_{m}: \mathbb{F}\left(n, m ; \mathbb{P}^{N}\right) \rightarrow \mathbb{G}\left(m, \mathbb{P}^{N}\right)$ be the natural projections. Then the following diagram on $\mathbb{F}\left(n, m ; \mathbb{P}^{N}\right)$ is commutative;

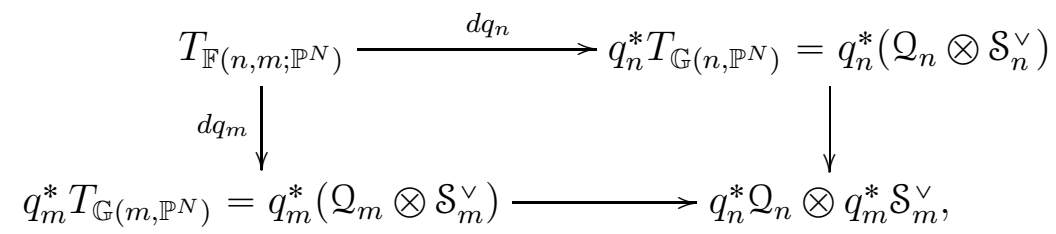

where the bottom and right maps are induced by the natural homomorphisms $q_{n}^{*} \mathcal{S}_{n}^{\vee} \rightarrow$ $q_{m}^{*} \mathcal{S}_{m}^{\vee}$ and $q_{m}^{*} Q_{m} \rightarrow q_{n}^{*} Q_{n}$ respectively. 
In particular, we obtain a commutative diagram with exact rows

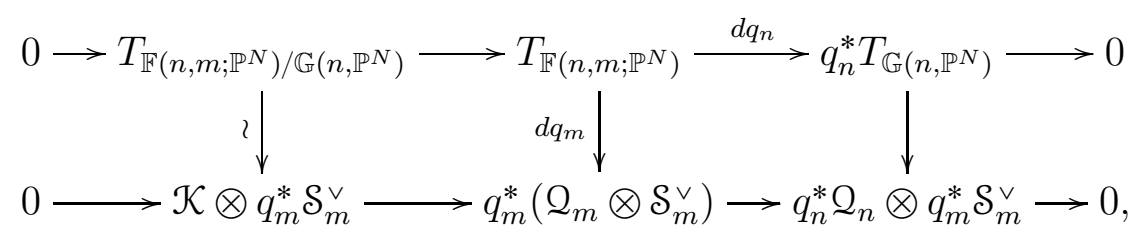

where $\mathcal{K}=\operatorname{ker}\left(q_{m}^{*} \mathcal{Q}_{m} \rightarrow q_{n}^{*} Q_{n}\right) \simeq q_{n}^{*} \mathcal{S}_{n} / q_{m}^{*} \mathcal{S}_{m}$.

Proof. The case when $n=0$ is nothing but [12, Lemma 2.6] ( $m$ corresponds to " $n$ " of that). As in the proof of that lemma, the commutativity of (2.4) can be checked by taking local coordinates on $\mathbb{P}^{N}$. We leave the detail to the reader.

We note that the left homomorphism in $(2.5)$ is an isomorphism since $\mathbb{F}\left(n, m ; \mathbb{P}^{N}\right)$ is nothing but the Grassmann bundle $G\left(N-m, \mathcal{S}_{n}^{\vee}\right)$ over $\mathbb{G}\left(n, \mathbb{P}^{N}\right)$.

Now return to the original setting. The sheaves

$$
\operatorname{pr}_{2}^{*}\left(\gamma_{n}^{*} Q_{n}^{\vee}\right) \subset \operatorname{pr}_{1}^{*} Q^{\vee} \subset H^{0}\left(\mathbb{P}^{N}, \mathcal{O}(1)\right)^{\vee} \otimes \mathcal{O}_{P_{m}^{X}}
$$

on $P_{m}^{X^{\circ}} \subset \mathbb{G}\left(m, \mathbb{P}^{N}\right) \times X^{\circ}$ define a morphism

$$
\left(\gamma_{n} \circ \mathrm{pr}_{2}, \mathrm{pr}_{1}\right): P_{m}^{X^{\circ}} \rightarrow \mathbb{F}\left(n, m ; \mathbb{P}^{N}\right) \quad: \quad(x, L) \mapsto\left(\mathbb{T}_{x} X, L\right) .
$$

We note that $P_{m}^{X^{\circ}}$ is the fiber product of $X^{\circ}$ and $\mathbb{F}\left(n, m ; \mathbb{P}^{N}\right)$ over $\mathbb{G}\left(n, \mathbb{P}^{N}\right)$ by the diagram

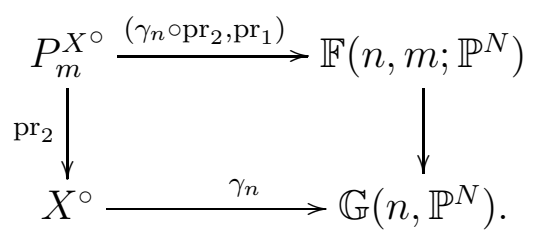

Lemma 2.8. The kernel of the differential $d \mathrm{pr}_{1}: T_{P_{m}^{x^{\circ}}} \rightarrow \operatorname{pr}_{1}^{*} T_{\mathbb{G}\left(m, \mathbb{P}^{N}\right)}$ is isomorphic to the kernel of $\varphi_{1}$ under $d \mathrm{pr}_{2}: T_{P_{m}^{X^{\circ}}} \rightarrow \operatorname{pr}_{2}^{*} T_{X^{\circ}}$.

Proof. By pulling back the diagram (2.5) by $\left(\gamma_{n} \circ \mathrm{pr}_{2}, \mathrm{pr}_{1}\right)$, we have a commutative diagram with exact rows

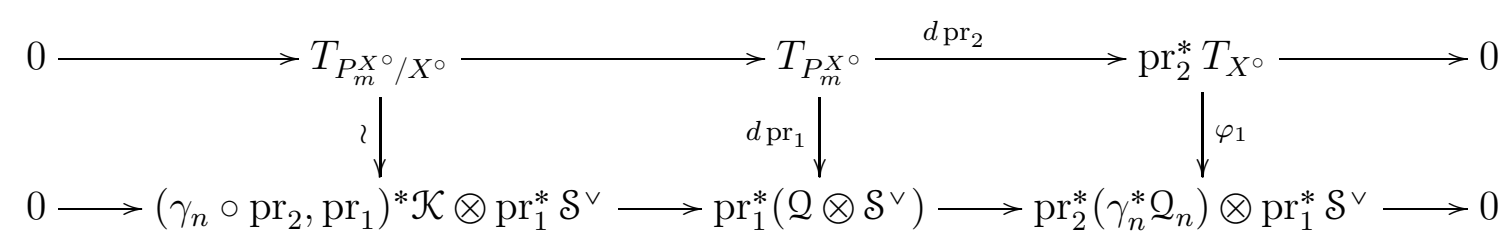

on $P_{m}^{X^{\circ}} \subset \mathbb{G}\left(m, \mathbb{P}^{N}\right) \times X^{\circ}$. We note that the left homomorphism is an isomorphism since the diagram (2.6) is Cartesian. Hence $\operatorname{ker} d \operatorname{pr}_{1} \simeq \operatorname{ker} \varphi_{1}$ holds. 
Lemma 2.9. For $\Phi=\Phi_{\operatorname{pr}_{1}}$, $\operatorname{ker} \Phi \subset \operatorname{pr}_{1}^{*} \mathcal{Q}^{\vee} \subset H^{0}\left(\mathbb{P}^{N}, \mathcal{O}(1)\right)^{\vee} \otimes \mathcal{O}_{P_{m}^{X}} \circ$ coincides with the kernel of the composite homomorphism

$$
\Phi^{\prime}: \operatorname{pr}_{2}^{*}\left(\gamma_{n}^{*} Q_{n}^{\vee}\right) \rightarrow \operatorname{pr}_{2}^{*} T_{X^{\circ}}(-1) \stackrel{\varphi_{2}(-1)}{\longrightarrow} \operatorname{pr}_{2}^{*} \Omega_{X^{\circ}} \otimes \operatorname{pr}_{1}^{*} \mathcal{S}^{\vee}
$$

as a subsheaf of $H^{0}\left(\mathbb{P}^{N}, \mathcal{O}(1)\right)^{\vee} \otimes \mathcal{O}_{P_{m}^{X}}$.

Proof. The diagram (2.5) induces

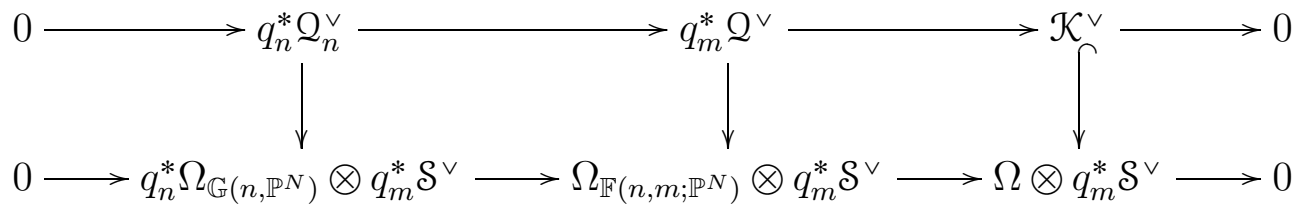

on $\mathbb{F}\left(n, m ; \mathbb{P}^{N}\right)$, where $\Omega=\Omega_{\mathbb{F}\left(n, m ; \mathbb{P}^{N}\right) / \mathbb{G}\left(n, \mathbb{P}^{N}\right)} \simeq \mathcal{K}^{\vee} \otimes q_{m}^{*} \mathcal{S}$. Since the natural homomorphism $\mathcal{O}_{\mathbb{F}\left(n, m ; \mathbb{P}^{N}\right)} \rightarrow q_{m}^{*}\left(\mathcal{S} \otimes \mathcal{S}^{\vee}\right)$ is injective, so is the right vertical homomorphism.

By pulling back the above diagram by $\left(\gamma_{n} \circ \mathrm{pr}_{2}, \mathrm{pr}_{1}\right)$, we obtain

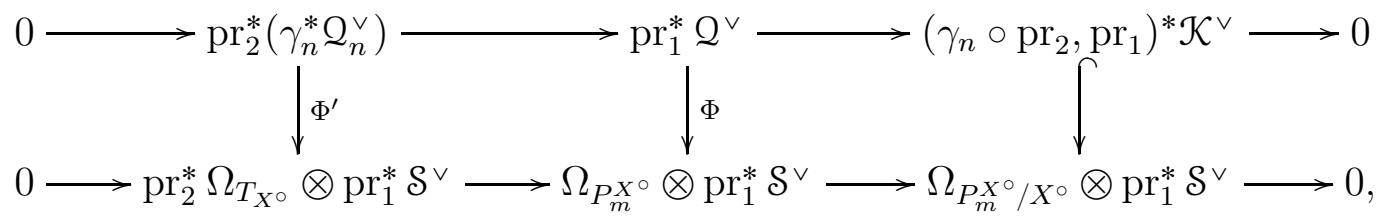

on $P_{m}^{X^{\circ}} \subset \mathbb{G}\left(m, \mathbb{P}^{N}\right) \times X^{\circ}$. We note that the middle homomorphism is nothing but $\Phi=\Phi_{\mathrm{pr}_{1}}$ and the left one is $\Phi^{\prime}$ in the statement of this lemma. Hence $\operatorname{ker} \Phi$ coincides with $\operatorname{ker} \Phi^{\prime}$ as a subsheaf of $H^{0}\left(\mathbb{P}^{N}, \mathcal{O}(1)\right)^{\vee} \otimes \mathcal{O}_{P_{m}^{X^{\circ}}}$.

Proof of Proposition 2.6. By Lemma 2.9, $\sigma_{P_{m}^{X}, \gamma_{m}}$ is induced by the kernel of the composition $\operatorname{pr}_{2}^{*}\left(\gamma_{n}^{*} Q_{n}^{\vee}\right) \rightarrow \operatorname{pr}_{2}^{*} T_{X^{\circ}}(-1) \stackrel{\varphi_{2}(-1)}{\longrightarrow} \operatorname{pr}_{2}^{*} \Omega_{X^{\circ}} \otimes \operatorname{pr}_{1}^{*} \mathcal{S}^{\vee}$. By Lemma 2.8, $\kappa_{m}$ is induced by the kernel of $\operatorname{pr}_{2}^{*}\left(\gamma_{n}^{*} Q_{n}^{\vee}\right) \rightarrow \operatorname{pr}_{2}^{*} T_{X^{\circ}}(-1) \stackrel{\varphi_{1}(-1)}{\longrightarrow} \operatorname{pr}_{2}^{*} \Omega_{X^{\circ}} \otimes \operatorname{pr}_{1}^{*} \mathcal{S}^{\vee}$. Since $\varphi_{1}=\varphi_{2}$, this proposition follows.

Proof of Theorem 2.2. (1) $\Rightarrow(2)$ : Let $\kappa_{m}: P_{m}^{X} \rightarrow \mathbb{G}\left(n_{\kappa}^{-}, \mathbb{P}^{N}\right)$ be the $m$-th degeneracy map, where $n_{\kappa}^{-}=\operatorname{rk}\left(\operatorname{ker} d \gamma_{m}\right)$ for $d \gamma_{m}: T_{P_{m}^{X}} \rightarrow \gamma_{m}^{*} T_{Y}$. Since $d \gamma_{m}$ is surjective, we have $n_{\kappa}^{-}=\operatorname{dim} P_{m}^{X}-\operatorname{dim} Y$. From Proposition 2.6, we have $\kappa_{m}=$ $\sigma_{P_{m}^{X}, \gamma_{m}}$. Since $\gamma_{m}$ is separable, [12, Remark 2.3] implies $\sigma_{P_{m}^{X}, \gamma_{m}}=\sigma_{Y} \circ \gamma_{m}$; hence $\kappa_{m}=\sigma_{Y} \circ \gamma_{m}$ and $n_{\kappa}^{-}=m_{\sigma_{Y}}^{-}=m^{-}$hold. Thus we have $m^{-}=\operatorname{dim} P_{m}^{X}-\operatorname{dim} Y$.

By Proposition 2.4, it holds that $P_{m}^{X} \subset \sigma_{Y}^{*} \mathcal{U}_{\mathbb{G}\left(m^{-}, \mathbb{P}^{N}\right)}$. Since

$$
\operatorname{dim} \sigma_{Y}^{*} \mathcal{U}_{\mathbb{G}\left(m^{-}, \mathbb{P}^{N}\right)}=\operatorname{dim} Y+m^{-}=\operatorname{dim} P_{m}^{X},
$$

$P_{m}^{X}$ coincides with $\sigma_{Y}^{*} \mathcal{U}_{\mathbb{G}\left(m^{-}, \mathbb{P}^{N}\right)}$. 
$(3) \Rightarrow(2)$ : Set $X^{\prime}=\sigma_{Y}^{*} \mathcal{U}_{\mathbb{G}\left(m^{-}, \mathbb{P}^{N}\right)}$. Since $\operatorname{pr}_{1}: X^{\prime} \rightarrow Y$ is a projective bundle, it is separable; hence $\sigma_{X^{\prime}, \mathrm{pr}_{1}}=\sigma_{Y} \circ \mathrm{pr}_{1}$ holds and then the condition (iii) of Proposition 2.4 is satisfied. Thus Proposition 2.4 implies (2).

The implications $(2) \Rightarrow(1)$ and $(2) \Rightarrow(3)$ follow immediately.

As we have already seen, Theorem 2.2 implies Theorem 1.1. In fact, we have the following result.

Corollary 2.10. Assume that $\gamma_{m}$ is separable. Let $\sigma_{X_{m}^{*}}=\sigma_{X_{m}^{*}, \iota}: X_{m}^{*} \rightarrow$ $\mathbb{G}\left(m^{-}, \mathbb{P}^{N}\right)$ be the shrinking map of $X_{m}$ with respect to $\iota: X_{m}^{*} \hookrightarrow \mathbb{G}\left(m, \mathbb{P}^{N}\right)$. Then $\sigma_{X_{m}^{*}}(L) \in \mathbb{G}\left(m^{-}, \mathbb{P}^{N}\right)$ corresponds to the contact locus $\pi\left(\gamma_{m}^{-1}(L)\right) \subset X$ for a general tangent $m$-plane $L \in X_{m}^{*}$.

Proof. As in the proof of Theorem 1.1, taking $Y=X_{m}^{*}$, we have the assertion from Theorem $2.2(2)$.

\section{Properties of $m$-Th DefeCts}

Let $X \subset \mathbb{P}^{N}$ be a non-degenerate projective variety of dimension $n$. For an integer $m$ with $n \leqslant m<N$, we write $\delta_{m}=\delta_{m}(X):=\operatorname{dim} P_{m}^{X}-\operatorname{dim} X_{m}^{*}$, the $m$-th defect of $X$. In this section, we do not assume the separability of $\gamma_{m}$.

We set $\sigma_{m}^{L}:=\pi\left(\gamma_{m}^{-1}(L)_{\text {red }}\right) \subset X$, the contact locus (1.1) of an tangent $m$-plane $L \in X_{m}^{*}$. Then $\delta_{m}=\operatorname{dim}\left(\sigma_{m}^{L}\right)$ for general $L \in X_{m}^{*}$. Note that $\sigma_{m}^{L} \subset X$ is equal to $\sigma_{X_{m}^{*}}(L)$ if $\gamma_{m}$ is separable (see Corollary 2.10).

E. Ballico showed the following statement for reflexive $X$ (see [1, Proposition $1])$. We show it in any case.

Lemma 3.1. It holds that $\delta_{m-1}+\delta_{m+1} \geqslant 2 \delta_{m}$ for $n<m<N-1$.

Proof. Let $\mathbb{F}\left(m_{1}, \ldots, m_{r} ; \mathbb{P}^{N}\right)$ be the flag variety parametrizing $\mathbb{P}^{m_{1}} \subset \mathbb{P}^{m_{2}} \subset \cdots \subset$ $\mathbb{P}^{m_{r}} \subset \mathbb{P}^{N}$, and let

$$
\begin{aligned}
\mathcal{V} & :=\mathbb{F}\left(m-1, m, m+1 ; \mathbb{P}^{N}\right) \times_{\mathbb{F}\left(m-1, m+1 ; \mathbb{P}^{N}\right)} \mathbb{F}\left(m-1, m, m+1 ; \mathbb{P}^{N}\right) \\
& =\left\{\left(M^{\prime}, L_{1}, L_{2}, M\right) \mid M^{\prime} \subset L_{i} \subset M \text { for } i=1,2\right\} \\
& \subset \mathbb{G}\left(m-1, \mathbb{P}^{N}\right) \times \mathbb{G}\left(m, \mathbb{P}^{N}\right) \times \mathbb{G}\left(m, \mathbb{P}^{N}\right) \times \mathbb{G}\left(m+1, \mathbb{P}^{N}\right) .
\end{aligned}
$$

Then

$$
\mathcal{V} \times_{\mathbb{G}\left(m-1, \mathbb{P}^{N}\right)} P_{m-1}^{X}=\overline{\left\{\left(M^{\prime}, L_{1}, L_{2}, M, x\right) \mid x \in X^{s m} \text { and } \mathbb{T}_{x} X \subset M^{\prime}\right\}}
$$

is irreducible. Take a general $\left(M^{\prime}, L_{1}, L_{2}, M, x\right) \in \mathcal{V} \times_{\mathbb{G}\left(m-1, \mathbb{P}^{N}\right)} P_{m-1}^{X}$. Then $\left(M^{\prime}, x\right),\left(L_{i}, x\right),(M, x)$ are general in $P_{m-1}^{X}, P_{m}^{X}, P_{m+1}^{X}$ respectively since projections

$$
\begin{aligned}
& \mathcal{V} \times_{\mathbb{G}\left(m-1, \mathbb{P}^{N}\right)} P_{m-1}^{X} \rightarrow P_{m-1}^{X}:\left(M^{\prime}, L_{1}, L_{2}, M, x\right) \mapsto\left(M^{\prime}, x\right) \\
& \mathcal{V} \times_{\mathbb{G}\left(m-1, \mathbb{P}^{N}\right)} P_{m-1}^{X} \rightarrow P_{m}^{X}:\left(M^{\prime}, L_{1}, L_{2}, M, x\right) \mapsto\left(L_{i}, x\right) \\
& \mathcal{V} \times_{\mathbb{G}\left(m-1, \mathbb{P}^{N}\right)} P_{m-1}^{X} \rightarrow P_{m+1}^{X}:\left(M^{\prime}, L_{1}, L_{2}, M, x\right) \mapsto(M, x)
\end{aligned}
$$


are surjective.

Hence $\sigma_{m-1}^{M^{\prime}}, \sigma_{m}^{L_{i}}, \sigma_{m+1}^{M}$ are smooth at $x$ of dimensions $\delta_{m-1}, \delta_{m}, \delta_{m+1}$ respectively. Since $\sigma_{m}^{L_{i}}$ is of codimension $r=\delta_{m+1}-\delta_{m}$ in $\sigma_{m+1}^{M}$ at $x$, the dimension $\delta_{m-1}$ of the intersection $\sigma_{m-1}^{M^{\prime}}=\sigma_{m}^{L_{1}} \cap \sigma_{m}^{L_{2}}$ at $x$ is at least $\geqslant \delta_{m}-r$.

Remark 3.2. It is known that the property of Lemma 3.1 induces the convexity, that is, for 4 integers $a, b, c, d$ with $a+d=b+c$ and $n \leqslant a<b \leqslant c<d \leqslant N-1$, it holds that $\delta_{a}+\delta_{d} \geqslant \delta_{b}+\delta_{c}$.

For smooth $X$, since $\delta_{n}=0$, the convexity gives the following corollaries.

Corollary 3.3. Assume that $X$ is smooth, and let $m$ be an integer with $n<m<$ $N-1$. Then the following holds.

(1) If $\delta_{m}>0$, then $\delta_{m+1}>\delta_{m}$.

(2) Assume $\delta_{m}>0$ and $\delta_{m+1}=\delta_{m}+1$. Then $\delta_{m-1}=\delta_{m}-1$. Moreover $\delta_{m-i}=\delta_{m}-i$ holds for all $0 \leqslant i \leqslant \delta_{m}$.

By a theorem of Zak [29, I, 2.3 Theorem], if $X \subset \mathbb{P}^{N}$ is smooth, then $\delta_{n+i} \leqslant i$ for all integer $i \geqslant 0$ with $n+i \leqslant N-1$. Hence we have:

Corollary 3.4. Assume that $X$ is smooth, and assume that $\delta_{n+i_{0}}=i_{0}$ holds for an integer $i_{0}>0$ with $n+i_{0}<N-1$. Then $\delta_{n+i}=i$ holds for all integer $i \geqslant 0$ with $n+i \leqslant N-1$. In particular, $\delta_{N-1}=N-n-1$, that is, $\operatorname{dim}\left(X^{*}\right)=n$.

Proof. It follows for $i>i_{0}$ due to Zak's theorem and Corollary 3.3 (1). It follows for $i<i_{0}$ by Corollary $3.3(2)$.

Lemma 3.5. Let $X \subset \mathbb{P}^{N}$ be a projective variety of dimension $n$, and let $k, m$ be integers with $0<k<n<m<N$. Let $A \subset \mathbb{P}^{N}$ be a $k$-plane contained in $X$ with $A \cap X^{s m} \neq \varnothing$, and let $L \subset \mathbb{P}^{N}$ be an m-plane tangent to $X$ at some point of $A \cap X^{s m}$. Then $\sigma_{m}^{L} \cap A$ is of dimension $\geqslant(N-m+1) k-(N-m) n$. In particular,

$$
\delta_{m} \geqslant(N-m+1) k-(N-m) n
$$

if $X$ is covered by $k$-planes.

Proof. Set $A^{\circ}=A \cap X^{s m}$. We consider

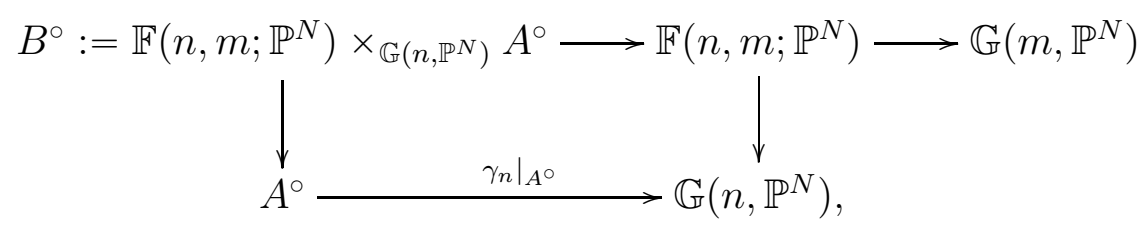

where $\left.\gamma_{n}\right|_{A^{\circ}}$ is the restriction of the Gauss map of $X$ on $A^{\circ}$. Since

$$
B^{\circ}=\left\{(L, a) \mid \mathbb{T}_{a} X \subset L\right\} \subset \mathbb{G}\left(m, \mathbb{P}^{N}\right) \times A^{\circ},
$$


we have $A \subset L$ for $(L, a) \in B^{\circ}$. Hence the image of $f: B \rightarrow \mathbb{G}\left(m, \mathbb{P}^{N}\right)$ is contained in $A_{m}^{*}=\left\{L \in \mathbb{G}\left(m, \mathbb{P}^{N}\right) \mid A \subset L\right\}$, where $B:=\overline{B^{\circ}} \subset P_{m}^{X}$. Since $\operatorname{dim}(B)=k+(m-n)(N-m)$ and $\operatorname{dim} f(B) \leqslant \operatorname{dim}\left(A_{m}^{*}\right)=(m-k)(N-m)$, each fiber of $B \rightarrow f(B)$ is of dimension $\geqslant k+(k-n)(N-m)$.

Example 3.6. Let $X \subset \mathbb{P}^{N}$ be a projective bundle over a smooth curve $C$ such that each fiber of $X \rightarrow C$ is a linear subvariety of $\mathbb{P}^{N}$. It is well known that $\delta_{N-1}=n-2$. The inequality $\delta_{N-1} \geqslant n-2$ follows from Lemma 3.5 since $X$ is covered by $(n-1)$-planes. The opposite inequality $\delta_{N-1} \leqslant n-2$ follows from [29, Chapter I, 2.3 Theorem b)].

Moreover, $\delta_{N-i} \geqslant n-1-i$ holds for each $1 \leqslant i \leqslant n-1$ by Lemma 3.5. By $\delta_{N-1}=n-2$ and Corollary 3.3, we have $\delta_{N-i}=n-1-i$ for $1 \leqslant i \leqslant n-1$.

Remark 3.7. Let $X \subset \mathbb{P}^{N}$ be a non-degenerate projective variety. Assume that the secant variety $S(X)$ is not equal to $\mathbb{P}^{N}$, and take a general point $z \in \mathbb{P}^{N}$ with $z \notin S(X)$. Let $\pi_{z}: \mathbb{P}^{N} \backslash\{z\} \rightarrow \mathbb{P}^{N-1}$ be the linear projection. Since $z \notin S(X)$, $\left.\pi_{z}\right|_{X}: X \rightarrow X_{z}:=\pi_{z}(X) \subset \mathbb{P}^{N-1}$ is isomorphic to the image $X_{z}$. In this setting, we have $\delta_{m}(X)=\delta_{m-1}\left(X_{z}\right)$ for $m>n$. The reason is as follows.

First, we note that for the contact locus $A \subset X$ of a general tangent $m$-plane $M$ with $z \in M$, we have $\operatorname{dim}(A)=\delta_{m}(X)$ since $z$ is general.

Let $M^{\prime} \subset \mathbb{P}^{N-1}$ be a $(m-1)$-plane, and set $M:=\pi_{z}^{-1}\left(M^{\prime}\right) \cup\{z\}$. Then $M^{\prime}$ is tangent to $X_{z}$ at a smooth point if and only if $M$ is tangent to $X$ at a smooth point. In such a case, the contact locus $A^{\prime} \subset X_{z}$ of $M^{\prime}$ coincides with $\pi_{z}(A)$, where $A \subset X$ is the contact locus of $M$. Hence if $M^{\prime}$ is a general $(m-1)$-plane tangent to $X_{z}$, it holds that $\delta_{m-1}\left(X_{z}\right)=\operatorname{dim}\left(A^{\prime}\right)=\operatorname{dim} A=\delta_{m}(X)$.

Example 3.8. Let $X^{\prime} \subset \mathbb{P}^{N^{\prime}}$ be a projective bundle over a smooth curve $C$ such that a general fiber of $X^{\prime} \rightarrow C$ is a linear variety in $\mathbb{P}^{N^{\prime}}$. Since $\operatorname{dim} S\left(X^{\prime}\right) \leqslant 2 n+1$, we can take $X \subset \mathbb{P}^{2 n+1}$ as the image of $X^{\prime}$ under $\mathbb{P}^{N^{\prime}} \rightarrow \mathbb{P}^{2 n+1}$, a composition of some linear projections, such that $X^{\prime} \simeq X$. Then $X$ satisfies $\delta_{n+3}=1$ as in Example 3.6.

Example 3.9. Let $X \subset \mathbb{P}^{2 n}$ be a projective bundle over a smooth elliptic curve such that each fiber is a linear variety in $\mathbb{P}^{2 n}$ (see [2, Corollary 2.3] for the existence of such $X$ ). Then $X$ satisfies $\delta_{n+2}=1$ as in Example 3.6.

Example 3.10. Let $X=\mathbb{P}^{1} \times \mathbb{P}^{n-1} \subset \mathbb{P}^{2 n-1}$, the Segre embedding. Then $X$ satisfies $\delta_{n+1}=1$; moreover $\delta_{n+i}=i$ for $i \geqslant 0$ as in Example 3.6.

\section{VARIETIES With POSITIVE $(n+1)$-TH DEFECT}

Let $X \subset \mathbb{P}^{N}$ be a non-degenerate projective variety of dimension $n$ over an algebraically closed field in arbitrary characteristic. In this section, we assume that $X$ is smooth and $n+2 \leqslant N-1$. Let $X_{m, x}^{*}:=\left\{L \in \mathbb{G}\left(m, \mathbb{P}^{N}\right) \mid \mathbb{T}_{x} X \subset L\right\} \subset X_{m}^{*}$, the set of $m$-planes tangent to $X$ at $x$. 
Definition 4.1. Let $x \in X$ and $L \in X_{m, x}^{*}$ be general. Then we can assume $x \in\left(\sigma_{m}^{L}\right)^{s m}$, and hence there exists a unique irreducible component of $\sigma_{m}^{L}$ containing $x$, which we denote by $\sigma_{m, x}^{L}$. By generality, we can also assume $\operatorname{dim}\left(\sigma_{m, x}^{L}\right)=\delta_{m}$.

Now we assume that $\gamma_{n+1}$ is separable and $\delta_{n+1}=1$. Then $\sigma_{n+1, x}^{L}=\sigma_{n+1}^{L}$ is a line by Theorem 1.1. From Corollary 3.4, we have $\delta_{n+2}=2$.

Lemma 4.2. Take a general $(M, x) \in P_{n+2}^{X}$. Then the unique irreducible component $\sigma_{n+2, x}^{M}$ of $\sigma_{n+2}^{M}$ containing $x$ is a surface covered by lines $\sigma_{n+1}^{L}$ 's with general $L \in X_{n+1, x}^{*}$ satisfying $L \subset M$.

Proof. Take a general $L \in X_{n+1, x}^{*}$ with $L \subset M$. Since $\sigma_{n+1}^{L} \subset \sigma_{n+2}^{M}$, the line $\sigma_{n+1}^{L}$ is contained in the surface $\sigma_{n+2, x}^{M}$. For two general $(n+1)$-planes $L, L^{\prime} \in X_{n+1, x}^{*}$ with $L, L^{\prime} \subset M$, we have

$$
\sigma_{n+1}^{L} \neq \sigma_{n+1}^{L^{\prime}}
$$

as follows. Suppose that the equality holds. Since $(n+1)$-planes $L, L^{\prime}$ contain $n$-plain $\mathbb{T}_{x} X$ and $L \neq L^{\prime}$, we have $L \cap L^{\prime}=\mathbb{T}_{x} X$. Since two lines $\sigma_{n+1}^{L}$ and $\sigma_{n+1}^{L^{\prime}}$ coincide, taking a general point $x^{\prime}$ of the line, we have $\mathbb{T}_{x^{\prime}} X \subset L \cap L^{\prime}=\mathbb{T}_{x} X$; thus $\mathbb{T}_{x^{\prime}} X=\mathbb{T}_{x} X$. This contradicts the finiteness of $\gamma_{n}$ for smooth $X$. $\sigma_{n+2, x}^{M}$.

Hence two lines $\sigma_{n+1}^{L}$ and $\sigma_{n+1}^{L^{\prime}}$ are distinct, and then such lines cover the surface

We denote by $\left(X_{n+1}^{*}\right)^{\circ}$ the set of $L \in X_{n+1}^{*}$ such that $\sigma_{n+1}^{L}$ is a line, and by $\left(P_{n+1}^{X}\right)^{\circ}$ the intersection $P_{n+1}^{X} \cap\left(\mathbb{P}^{N} \times\left(X_{n+1}^{*}\right)^{\circ}\right)$. Let us consider

$$
\begin{gathered}
P_{n+1}^{X} \times_{X_{n+1}^{*}} P_{n+1}^{X} \simeq\left\{\left(L, x, x^{\prime}\right) \in \mathbb{G}\left(n+1, \mathbb{P}^{N}\right) \times X \times X \mid \mathbb{T}_{x} X, \mathbb{T}_{x^{\prime}} X \subset L\right\} \\
\left(P_{n+1}^{X}\right)^{\circ} \times_{X_{n+1}^{*}}\left(P_{n+1}^{X}\right)^{\circ} \subset P_{n+1}^{X} \times_{X_{n+1}^{*}} P_{n+1}^{X} \rightarrow X \times X
\end{gathered}
$$

and set

$$
\Lambda^{\circ} \subset X \times X
$$

to be the image of the morphism (4.2). Let $\Lambda \subset X \times X$ be the closure of $\Lambda^{\circ}$, where the $i$-th projection $\rho_{i}: \Lambda \rightarrow X$ with $i=1,2$ is separable since so is $\gamma_{n+1}$.

We denote by $\Lambda_{x}=\rho_{2}\left(\rho_{1}^{-1}(x)\right) \subset X$. As a set, $\Lambda_{x}$ can be described as follows: For general $x \in X$, set $\left(X_{n+1, x}^{*}\right)^{\circ}=X_{n+1, x}^{*} \cap\left(X_{n+1}^{*}\right)^{\circ}$. Then the fiber of

$$
\left(P_{n+1}^{X}\right)^{\circ} \times_{X_{n+1}^{*}}\left(P_{n+1}^{X}\right)^{\circ} \subset P_{n+1}^{X} \times_{X_{n+1}^{*}} P_{n+1}^{X} \rightarrow X:\left(L, x, x^{\prime}\right) \mapsto x
$$

over general $x \in X$ is

$$
\left\{\left(L, x^{\prime}\right) \in\left(X_{n+1, x}^{*}\right)^{\circ} \times X \mid x^{\prime} \in \sigma_{n+1}^{L}\right\} .
$$


Hence the fiber $\Lambda_{x}^{\circ}$ over $x$ of the first projection $\Lambda^{\circ} \rightarrow X$ is

$$
\bigcup_{L \in\left(X_{n+1, x}^{*}\right)^{\circ}} \sigma_{n+1}^{L}=\pi\left(\gamma_{n+1}^{-1}\left(X_{n+1, x}^{*}\right)^{\circ}\right) \subset X
$$

and $\Lambda_{x}$ is the closure of this set. We note that for general $M \in X_{n+2, x}^{*}$, we have $\sigma_{n+2, x}^{M} \subset \Lambda_{x}$ by Lemma 4.2 since $(x, M) \in P_{n+2, x}^{X}$ is general.

Remark 4.3. (1) We set

$$
\sigma:=\sigma_{X_{n+1}^{*}}: X_{n+1}^{*} \rightarrow \mathbb{G}\left(1, \mathbb{P}^{N}\right)
$$

to be the shrinking map of $X_{n+1}^{*}$ with respect to $\iota: X_{n+1}^{*} \hookrightarrow \mathbb{G}\left(n+1, \mathbb{P}^{N}\right)$. Then $\sigma(L)=\sigma_{n+1}^{L}$ for general $L \in X_{n+1}^{*}$ as in Corollary 2.10.

(2) $\Lambda_{x}$ is an irreducible cone with vertex $x$. The reason is as follows. It is irreducible since $\Lambda_{x}^{\circ}$ coincides with the image of

$$
\left.\mathcal{U}_{\mathbb{G}\left(1, \mathbb{P}^{N}\right)}\right|_{\sigma\left(\left(X_{n+1, x}^{*}\right)^{\circ}\right)} \subset \mathbb{G}\left(1, \mathbb{P}^{N}\right) \times X
$$

under the second projection. In addition, it is a cone with vertex $x$ since each $\sigma_{n+1}^{L}$ is a line containing $x$.

(3) Each fiber of $P_{n+1}^{X} \times_{X_{n+1}^{*}} P_{n+1}^{X} \rightarrow P_{n+1}^{X}$ at $(L, x) \in P_{n+1}^{X}$ corresponds to $\sigma_{n+1}^{L}$, whose dimension is $\delta_{n+1}=1$ if $(x, L)$ is general. Since $\operatorname{dim}\left(P_{n+1}^{X}\right)=N-1$, we have $P_{n+1}^{X} \times_{X_{n+1}^{*}} P_{n+1}^{X}=N$.

Lemma 4.4. $\operatorname{dim} \Lambda_{x}=N-n$ for general $x \in X$.

Proof. Let $\sigma$ be the shrinking map as in Remark 4.3. Let $L_{F}:=\left\langle\bigcup_{x \in F} \mathbb{T}_{x} X\right\rangle \subset \mathbb{P}^{N}$ be the linear variety spanned by $\bigcup_{x \in F} \mathbb{T}_{x} X$ for general $F \in \sigma\left(X_{n+1}^{*}\right)$. Since $X$ is smooth, $\bigcup_{x \in F} \mathbb{T}_{x} X$ is of dimension $\geqslant n+1$, and so is $L_{F}$. Taking $L \in X_{n+1}^{*}$ such that $F=\sigma(L)=\pi\left(\gamma_{n+1}^{-1}(L)\right)$, we have $L_{F} \subset L$ and then $L_{F}=L$. In particular, $L_{F}$ is an $(n+1)$-plane. Therefore

$$
\sigma^{-1}(F)=\left\{L \in \mathbb{G}\left(n+1, \mathbb{P}^{N}\right) \mid L_{F} \subset L\right\}=\left\{L_{F}\right\} .
$$

In particular, $\sigma$ is generically injective.

Now we show that the morphism

$$
f:\left(P_{n+1}^{X}\right)^{\circ} \times_{X_{n+1}^{*}}\left(P_{n+1}^{X}\right)^{\circ} \rightarrow \Lambda^{\circ}
$$

given by (4.2) is generically bijective, as follows. A point of the left hand side is expressed by $\left(L, x, x^{\prime}\right)$ as in (4.1). For general $\left(x, x^{\prime}\right) \in \Lambda^{\circ}$, we take a point $\left(L, x, x^{\prime}\right) \in f^{-1}\left(x, x^{\prime}\right)$. Then, since $x, x^{\prime}$ are points of the line $\sigma(L)$, we have $\sigma(L)=$ $\overline{x x^{\prime}}$. It means that $f^{-1}\left(x, x^{\prime}\right) \simeq \sigma^{-1}\left(\overline{x x^{\prime}}\right) \times\left\{\left(x, x^{\prime}\right)\right\}$, which is indeed equal to the set of a point $\left(L \overline{x x^{\prime}}, x, x^{\prime}\right) \in \mathbb{G}\left(n+1, \mathbb{P}^{N}\right) \times X \times X$ because of (4.4).

Therefore $\operatorname{dim}\left(\Lambda^{\circ}\right)=\operatorname{dim}\left(\left(P_{n+1}^{X}\right)^{\circ} \times_{X_{n+1}^{*}}\left(P_{n+1}^{X}\right)^{\circ}\right)=N$ (see Remark 4.3). It follows that a general fiber of $\Lambda^{\circ} \rightarrow X$ is of dimension $N-n$. 
Lemma 4.5. Let $x \in X$ be a general point. Then $\Lambda_{x}=\Lambda_{x^{\prime}}$ for general $x^{\prime} \in \Lambda_{x}$. Therefore $\Lambda_{x}$ is scheme-theoretically a linear variety of $\mathbb{P}^{N}$.

Proof. Let $x \in X$ and $x^{\prime} \in \Lambda_{x}$ be general points as in the statement of this proposition. In other word, take general $\left(x, x^{\prime}\right) \in \Lambda^{\circ}$. By definition, there exists an $(n+1)$-plane $L$ such that $\left(L, x, x^{\prime}\right)$ is a general point in $\left(P_{n+1}^{X}\right)^{\circ} \times_{X_{n+1}^{*}}\left(P_{n+1}^{X}\right)^{\circ}$.

Take general $K \in X_{n+1, x}^{*}$ and set $M=\langle L, K\rangle$, where $M$ is an $(n+2)$-plane since $L \cap K=\mathbb{T}_{x} X$. Then $(x, M),\left(x^{\prime}, M\right) \in P_{n+2}^{X}$ are general, which will be shown later.

Hence we have the unique irreducible component $\sigma_{n+2, x}^{M}\left(\right.$ resp. $\left.\sigma_{n+2, x^{\prime}}^{M}\right)$ of $\sigma_{n+2}^{M}$ containing $x$ (resp. $\left.x^{\prime}\right)$. Since $L, K \subset M$, the lines $\sigma_{n+1}^{L}, \sigma_{n+1}^{K}$ are contained in $\sigma_{n+2}^{M}$. Hence $\sigma_{n+1}^{L}, \sigma_{n+1}^{K}$ must be contained in $\sigma_{n+2, x}^{M}$ because of $x \in \sigma_{n+1}^{L}, \sigma_{n+1}^{K}$. Since $x^{\prime} \in \sigma_{n+1}^{L}, x^{\prime}$ is also contained in $\sigma_{n+2, x}^{M}$. By the uniqueness of $\sigma_{n+2, x^{\prime}}^{M}$, we have $\sigma_{n+2, x^{\prime}}^{M}=\sigma_{n+2, x}^{M}$. Hence it follows from Lemma 4.2 that $\Lambda_{x^{\prime}} \supset \sigma_{n+2, x^{\prime}}^{M}=$ $\sigma_{n+2, x}^{M} \supset \sigma_{n+1}^{K}$. Recall that $K \in X_{n+1, x}^{*}$ can be any general element. Hence we have

$$
\Lambda_{x^{\prime}} \supset \bigcup_{K \in X_{n+1, x}^{*}, \text { general }} \sigma_{n+1}^{K}=\Lambda_{x} .
$$

Since $\Lambda_{x^{\prime}}, \Lambda_{x}$ have the same dimension, $\Lambda_{x^{\prime}}=\Lambda_{x}$ holds.

As a result, $\Lambda_{x}$ is a cone with vertex $x^{\prime}$ for general $x^{\prime} \in \Lambda_{x}$, because of $\Lambda_{x}=\Lambda_{x^{\prime}}$ and Remark 4.3. This implies that $\Lambda_{x}$ is a linear variety. We note that $\Lambda_{x}$ is reduced since $\rho_{1}: \Lambda \rightarrow X$ is separable.

To finish the proof, it suffices to check that for general $\left(L, x, x^{\prime}\right) \in\left(P_{n+1}^{X}\right)^{\circ} \times_{X_{n+1}^{*}}$ $\left(P_{n+1}^{X}\right)^{\circ}$ and general $K \in X_{n+1, x}^{*},(\langle L, K\rangle, x),\left(\langle L, K\rangle, x^{\prime}\right) \in P_{n+1}^{X}$ are also general elements.

To parametrize $\left(L, x, x^{\prime}\right) \in\left(P_{n+1}^{X}\right)^{\circ} \times_{X_{n+1}^{*}}\left(P_{n+1}^{X}\right)^{\circ}$ and $K \in X_{n+1, x}^{*}$, consider

$$
\begin{aligned}
P_{n+1}^{X} \times_{X}\left(\left(P_{n+1}^{X}\right)^{\circ} \times_{X_{n+1}^{*}}\left(P_{n+1}^{X}\right)^{\circ}\right) & =\left\{\left(K, L, x, x^{\prime}\right) \mid \mathbb{T}_{x} X, \mathbb{T}_{x^{\prime}} X \subset L, \mathbb{T}_{x} X \subset K\right\} \\
& \subset X_{n+1}^{*} \times\left(X_{n+1}^{*}\right)^{\circ} \times X \times X \\
& \subset \mathbb{G}\left(m, \mathbb{P}^{N}\right) \times \mathbb{G}\left(m, \mathbb{P}^{N}\right) \times X \times X,
\end{aligned}
$$

which is the fiber product of the projection $\left(P_{n+1}^{X}\right)^{\circ} \times_{X_{n+1}^{*}}\left(P_{n+1}^{X}\right)^{\circ} \rightarrow X:\left(L, x, x^{\prime}\right) \mapsto$ $x$ and $P_{n+1}^{X} \rightarrow X$.

For general $\left(K, L, x, x^{\prime}\right) \in P_{n+1}^{X} \times_{X}\left(\left(P_{n+1}^{X}\right)^{\circ} \times_{X_{n+1}^{*}}\left(P_{n+1}^{X}\right)^{\circ}\right),\langle K, L\rangle$ is an $(n+2)$ plane which contains $\mathbb{T}_{x} X, \mathbb{T}_{x^{\prime}} X$. Hence we have rational maps

$$
\begin{aligned}
& f: P_{n+1}^{X} \times_{X}\left(\left(P_{n+1}^{X}\right)^{\circ} \times_{X_{n+1}^{*}}\left(P_{n+1}^{X}\right)^{\circ}\right) \rightarrow P_{n+2}^{X}:\left(K, L, x, x^{\prime}\right) \mapsto(\langle K, L\rangle, x) \\
& f^{\prime}: P_{n+1}^{X} \times_{X}\left(\left(P_{n+1}^{X}\right)^{\circ} \times_{X_{n+1}^{*}}\left(P_{n+1}^{X}\right)^{\circ}\right)-\rightarrow P_{n+2}^{X}:\left(K, L, x, x^{\prime}\right) \mapsto\left(\langle K, L\rangle, x^{\prime}\right) .
\end{aligned}
$$

The rest is to see that $f, f^{\prime}$ are dominant. 
For general $(M, x) \in P_{n+2}^{X}$, take general $(n+1)$-planes $K, L \subset M$ containing $\mathbb{T}_{x} X$. Since $(M, x) \in P_{n+2}^{X}$ is general, we have $(K, x),(L, x) \in\left(P_{n+1}^{X}\right)^{\circ}$. For $x^{\prime} \in \sigma_{n+1}^{L}$, $\left(K, L, x, x^{\prime}\right)$ is an element in $P_{n+1}^{X} \times_{X}\left(\left(P_{n+1}^{X}\right)^{\circ} \times_{X_{n+1}^{*}}\left(P_{n+1}^{X}\right)^{\circ}\right)$ and $f\left(K, L, x, x^{\prime}\right)=$ $(M, x)$. Hence $f$ is dominant.

For general $\left(M, x^{\prime}\right) \in P_{n+2}^{X}$, take general $(n+1)$-planes $L \subset M$ which contains $\mathbb{T}_{x^{\prime}} X$. Since $\left(M, x^{\prime}\right) \in P_{n+2}^{X}$ is general, we have $\left(L, x^{\prime}\right) \in\left(P_{n+1}^{X}\right)^{\circ}$. Take $x \in \sigma_{n+1}^{L}$. Then $(L, x)$ is in $\left(P_{n+1}^{X}\right)^{\circ}$. For a general $(n+1)$-plane $K$ which contains $\mathbb{T}_{x} X$, $\left(K, L, x, x^{\prime}\right)$ is an element in $P_{n+1}^{X} \times_{X}\left(\left(P_{n+1}^{X}\right)^{\circ} \times_{X_{n+1}^{*}}\left(P_{n+1}^{X}\right)^{\circ}\right)$ and $f^{\prime}\left(K, L, x, x^{\prime}\right)=$ $\left(M, x^{\prime}\right)$. Hence $f^{\prime}$ is dominant.

Lemmas 4.4 and 4.5 imply the following result.

Proposition 4.6. Assume that $\gamma_{n+1}$ is separable and $\delta_{n+1}=1$. Then the first projection $\Lambda \rightarrow X$ of (4.3) induces a rational map

$$
X \rightarrow \mathbb{G}\left(N-n, \mathbb{P}^{N}\right)
$$

sending $x \mapsto \Lambda_{x}$. Let $Y \subset \mathbb{G}\left(N-n, \mathbb{P}^{N}\right)$ be the closure of the image of this rational map. For the universal family $\mathcal{U}_{Y} \subset Y \times \mathbb{P}^{N}$, the second projection $\mathcal{U}_{Y} \rightarrow \mathbb{P}^{N}$ is birational onto $X \subset \mathbb{P}^{N}$.

Proof. Since $\Lambda_{x}$ is a linear variety of dimension $N-n$ for general $x$, the rational map $X \rightarrow \mathbb{G}\left(N-n, \mathbb{P}^{N}\right)$ is obtained. We consider the graph $g: X \rightarrow Y \times \mathbb{P}^{N}$, which sends $x \mapsto\left(\Lambda_{x}, x\right)$. Since $x \in \Lambda_{x}$, the image of $g$ is contained in $\mathcal{U}_{Y}$.

In fact, $g$ is dominant to $\mathcal{U}_{Y}$. This is because, for general $\left(\Lambda, x^{\prime}\right) \in \mathcal{U}_{Y}$, we can write $\Lambda=\Lambda_{x}$ for some $x$, and then $\Lambda_{x}=\Lambda_{x^{\prime}}$ by Lemma 4.5, which implies that $g\left(x^{\prime}\right)=\left(\Lambda, x^{\prime}\right)$.

Since the composite map $X \rightarrow \mathcal{U}_{Y} \rightarrow X$ is identity, $\mathcal{U}_{Y} \rightarrow X$ is birational.

In fact, we have:

Proposition 4.7. In Proposition 4.6, $\mathcal{U}_{Y} \rightarrow X$ is an isomorphism.

Proof. Step 1. First we show that $\Lambda \subset \pi\left(\gamma_{n+1}^{-1}\left(X_{n+1, x}^{*}\right)\right)$ holds for any $(\Lambda, x) \in \mathcal{U}_{Y}$ as follows. Let us define a morphism

$$
\mathcal{U}_{Y} \times_{Y} \mathcal{U}_{Y} \simeq\left\{\left(\Lambda, x_{1}, x_{2}\right) \in Y \times \mathbb{P}^{N} \times \mathbb{P}^{N} \mid x_{1}, x_{2} \in \Lambda\right\} \rightarrow \mathbb{G}\left(n, \mathbb{P}^{N}\right) \times \mathbb{G}\left(n, \mathbb{P}^{N}\right)
$$

by $\left(\Lambda, x_{1}, x_{2}\right) \mapsto\left(\mathbb{T}_{x_{1}} X, \mathbb{T}_{x_{2}} X\right)$, and define $W$ to be the set of $\left(T_{1}, T_{2}\right) \in \mathbb{G}\left(n, \mathbb{P}^{N}\right) \times$ $\mathbb{G}\left(n, \mathbb{P}^{N}\right)$ such that $\operatorname{dim}\left\langle T_{1}, T_{2}\right\rangle \leqslant n+1$. Note that $W$ is a closed subset of $\mathbb{G}\left(m, \mathbb{P}^{N}\right) \times \mathbb{G}\left(m, \mathbb{P}^{N}\right)$.

In the above setting, the image of (4.5) is contained in $W$, as follows: A general member of the left hand side of $(4.5)$ is written as $\left(\Lambda_{x}, x, x^{\prime}\right)$ with general points $x \in$ $X$ and $x^{\prime} \in \Lambda_{x}$. By definition, $x^{\prime}$ is contained in $\sigma_{n+1}(L)=\pi\left(\gamma_{n+1}^{-1}(L)\right)$ with some 
$(n+1)$-plane $L \in\left(X_{n+1, x}^{*}\right)^{\circ}$. This means that $\mathbb{T}_{x^{\prime}} X \subset L$. Since $\left\langle\mathbb{T}_{x} X, \mathbb{T}_{x^{\prime}} X\right\rangle \subset L$, we have the assertion.

Take $(\Lambda, x) \in \mathcal{U}_{Y}$. For any $x^{\prime} \in \Lambda$, we have $\operatorname{dim}\left\langle\mathbb{T}_{x} X, \mathbb{T}_{x^{\prime}} X\right\rangle \leqslant n+1$ since (4.5) maps $\left(\Lambda, x, x^{\prime}\right)$ into $W$. Let $L$ be an $(n+1)$-plane containing $\left\langle\mathbb{T}_{x} X, \mathbb{T}_{x^{\prime}} X\right\rangle$. Then $\left(L, x^{\prime}\right) \in P_{n+1}^{X}$ and $L \in X_{n+1, x}^{*}$, which means that $x^{\prime} \in \pi\left(\gamma_{n+1}^{-1}\left(X_{n+1, x}^{*}\right)\right)$. Thus $\Lambda \subset \pi\left(\gamma_{n+1}^{-1}\left(X_{n+1, x}^{*}\right)\right)$ holds.

Step 2. Next we show the finiteness of $\mathcal{U}_{Y} \rightarrow X$. Fix $x \in X$ to be any point. By a theorem of Zak [29, I, 2.3 Theorem], every fiber of $\gamma_{n+1}: P_{n+1}^{X} \rightarrow X_{n+1}^{*}$ is of dimension $\leqslant 1$. Therefore every irreducible component of $\gamma_{n+1}^{-1}\left(X_{n+1, x}^{*}\right)$ is of dimension $\leqslant(N-n-1)+1=N-n$. From Step 1, every $(\Lambda, x) \in \mathcal{U}_{Y}$ satisfies $\Lambda \subset \gamma_{n+1}^{-1}\left(X_{n+1, x}^{*}\right)$. Since $\operatorname{dim}(\Lambda)=N-n$, in fact $\Lambda$ coincides with an irreducible component of $\gamma_{n+1}^{-1}\left(X_{n+1, x}^{*}\right)$. Hence there only exist finitely many $\Lambda$ 's such that $(\Lambda, x) \in \mathcal{U}_{Y}$. It means that $\mathcal{U}_{Y} \rightarrow X$ is finite.

By Zariski's main theorem, we find that $\mathcal{U}_{Y} \rightarrow X$ is isomorphic since it is birational by Proposition 4.6 and $X$ is smooth.

By Zak's inequality, we know $\delta_{n+i} \leqslant i$ for smooth $X \subset \mathbb{P}^{N}$. We study the case when the equality holds. The following theorem implies Theorem 1.2

Theorem 4.8. Let $X \subset \mathbb{P}^{N}$ be a non-degenerate smooth projective variety with $n:=\operatorname{dim}(X)<N-2$. Assume that $\gamma_{n+1}$ is separable. Then the following conditions are equivalent:

(a) $\delta_{n+i}=i$ holds for an integer $i>0$ with $n+i<N-1$.

(b) $\delta_{n+i}=i$ holds for any integer $i \geqslant 0$ with $n+i \leqslant N-1$.

(c) $X$ is the image of the Segre embedding $\mathbb{P}^{1} \times \mathbb{P}^{n-1} \hookrightarrow \mathbb{P}^{2 n-1}$.

Remark 4.9. Assume that the characteristic is zero. Then Ein [3, Theorem 3.4] showed that if $\operatorname{dim}(X)=N-2$ and $\delta_{N-1}\left(=\delta_{n+1}\right)=1$, then $X=\mathbb{P}^{1} \times \mathbb{P}^{2} \subset \mathbb{P}^{5}$. Hence combining with Theorem 4.8 , we have that $\mathbb{P}^{1} \times \mathbb{P}^{n-1} \subset \mathbb{P}^{2 n-1}$ is only the smooth projective variety of codimension $\geqslant 2$ which satisfies $\delta_{n+i}=i$ for all $i$ with $0 \leqslant i \leqslant N-n-1$.

Proof of Theorem 4.8. (c) $\Rightarrow$ (b) follows from Example 3.10. (b) $\Rightarrow$ (a) follows immediately. We show (a) $\Rightarrow$ (c) as follows.

From Corollary 3.4, we have $\delta_{n+1}=1$. From Proposition 4.7, $X$ is isomorphic to the projective bundle $\mathcal{U}_{Y}$ with $\operatorname{dim}(Y)=2 n-N$. In particular, $\operatorname{Pic}(X)$ is of rank $\geqslant 2$.

If $n \geqslant(N+2) / 2$, then $\operatorname{Pic}(X)=\mathbb{Z}$ if the characteristic $p=0$ due to the BarthLarsen theorem (see [23, Corollary 3.2.3] for example) and $\operatorname{Pic}(X)$ is a finitely generated abelian group of rank 1 if $p>0$ due to [26, Theorem (3.1)], respectively. Therefore $n<(N+2) / 2$ must hold, which implies $\operatorname{dim}(Y)=1$ and $N=2 n-1$. 
Then we find that the isomorphism $\mathcal{U}_{Y} \rightarrow X \subset \mathbb{P}^{2 n-1}$ is in fact the Segre embedding; see [20, pp. 307-308] or [25, Proposition 3.1].

Proof of Theorem 1.2. Assume that $\gamma_{n+1}$ is separable. If $X$ is not the Segre embedding, then it follows from Theorem 4.8 that $\delta_{n+1}=0$ must hold. Since the contact locus $\pi\left(\gamma_{n+1}^{-1}(L)\right) \subset X$ of general $L \in X_{n+1}^{*}$ is a linear variety because of Theorem 1.1, a general fiber is a point. This means that $\gamma_{n+1}$ is birational.

\section{REFERENCES}

[1] E. Ballico, On the Gauss maps of singular projective varieties, J. Austral. Math. Soc. 72 (2002), 119-130.

[2] C. Ciliberto and K. Hulek, A bound on the irregularity of abelian scrolls in projective space, Bauer, Ingrid (ed.) et al., Complex geometry. Collection of papers dedicated to Hans Grauert on the occasion of his 70th birthday. Berlin: Springer, 2002, pp. 85-92

[3] L. Ein, Varieties with small dual varieties, I, Invent. Math., 86 (1986), 63-74

[4] _ Varieties with small dual varieties, II, Duke. Math. J., 52 (1985), 895-907.

[5] G. Fischer and J. Piontkowski, Ruled varieties, Advanced Lectures in Mathematics, Friedr. Vieweg \& Sohn, Braunschweig, 2001.

[6] S. Fukasawa, Developable varieties in positive characteristic. Hiroshima Math. J. 35 (2005), $167-182$.

[7] _ - Varieties with non-linear Gauss fibers. Math. Ann. 334 (2006), 235-239.

[8] _ On Kleiman-Piene's question for Gauss maps. Compositio Math. 142 (2006), 13051307.

[9] , A remark on Kleiman-Piene's question for Gauss maps. Comm. Algebra 35 (2007), 1201-1204.

[10] K. Furukawa, Duality with expanding maps and shrinking maps, and its applications to Gauss maps, Math. Ann. 358 (2014), 403-432.

[11] K. Furukawa and A. Ito, Gauss maps of toric varieties, arXiv:1403.0793, to appear in Tohoku Math. J.

[12] _ On Gauss maps in positive characteristic in view of images, fibers, and field extensions, to appear in IMRN, doi: 10.1093/imrn/rnw080.

[13] P. Griffiths and J. Harris, Algebraic geometry and local differential geometry, Ann. Sci. Ecole Norm. Sup. (4) 12 (1979), 355-432.

[14] A. Hefez and S. Kleiman, Notes on the duality of projective varieties. Geometry today (Rome, 1984), 143183, Progr. Math. 60, pp. 143-183. Birkhaüser Boston, Boston, MA, 1985.

[15] T. A. Ivey and J. M. Landsberg, Cartan for beginners: differential geometry via moving frames and exterior differential systems, Grad. Stud. Math. 61, Amer. Math. Soc., Providence, 2003.

[16] H. Kaji, On the tangentially degenerate curves. J. London Math. Soc. (2) 33 (1986), 430440.

[17] _ On the Gauss maps of space curves in characteristic $p$. Compositio Math. 70 (1989), $177-197$.

[18] _ On the duals of Segre varieties. Geom. Dedicata 99 (2003), 221-229.

[19] _ Higher Gauss maps of Veronese varieties -A generalization of Boole's formula-, arXiv:1509.04935. 
[20] S. L. Kleiman, Plane forms and multiple-point formulas, in 'Algebraic threefolds (Varenna, 1981)', Lecture Notes in Math., 947, Springer, Berlin, 1982, pp. 287-310.

[21] _ Tangency and duality, "Proceedings of the 1984 Vancouver conference in algebraic geometry", CMS Conference Proceedings 6, Amer. Math. Soc., Providence, 1986, pp.163226.

[22] S. L. Kleiman and R. Piene, On the inseparability of the Gauss map. "Enumerative Algebraic Geometry (Proceedings of the 1989 Zeuthen Symposium)," Contemp. Math. 123, pp. 107129. Amer. Math. Soc., Providence, 1991.

[23] R. Lazarsfeld, Positivity in algebraic geometry I, Ergebnisse der Mathematik undihrer Grenzgebiete, vol. 48. Springer, Berlin (2004).

[24] A. Noma, Gauss maps with nontrivial separable degree in positive characteristic. J. Pure Appl. Algebra 156 (2001), 81-93.

[25] J. C. Sierra, A remark on zak's theorem on tangencies. Math. Res. Lett. 18 (2011), 783-789.

[26] R. Speiser, Vanishing criteria and the Picard group for projective varieties of low codimension. Compositio Math., 42 (1981), 13-21.

[27] J. Rathmann, The uniform position principle for curves in characteristic $p$. Math. Ann. 276 (1987), 565-579.

[28] A. H. Wallace, Tangency and duality over arbitrary fields. Proc. London Math. Soc. (3) 6 (1956), 321-342.

[29] F. L. Zak, Tangents and secants of algebraic varieties. Transl. Math. Monographs 127, Amer. Math. Soc., Providence, 1993.

Graduate School of Mathematical Sciences, the University of Tokyo, Tokyo, JAPAN

E-mail address: katu@ms.u-tokyo.ac.jp

Department of Mathematics, Kyoto University, Kyoto, Japan

E-mail address: aito@math.kyoto-u.ac.jp 